\title{
NISTIR 6205
}

\section{Neutron Time-of-Flight Spectroscopy}

ac 100 .456 i. 0.6205 1998
United States Department of Commerce Technology Administration

National Institute of Standards and Technology 



\section{NISTIR 6205}

\section{Neutron Time-of-Flight Spectroscopy}

John R. D. Copley

NIST Center for Neutron Research

Materials Science and Engineering Laboratory

National Institute of Standards and Technology

August 1998

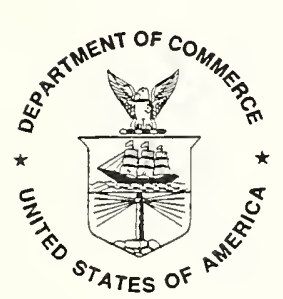

U.S. DEPARTMENT OF COMMERCE

William M. Daley, Secretary

TECHNOLOGY ADMINISTRATION

Gary R. Bachula, Acting Under Secretary for Technology

NATIONAL INSTITUTE OF STANDARDS AND TECHNOLOGY

Raymond G. Kammer, Director 


\title{
Neutron Time-of-Flight Spectroscopy
}

\author{
John R.D. Copley, \\ NIST Center for Neutron Research, \\ National Institute of Standards and Technology, \\ Gaithersburg, MD 20899, USA
}

In this lecture we shall discuss the technique of time-of-flight spectroscopy. Strictly speaking, all experiments performed using instruments at a pulsed neutron source can be characterized as time-of-flight spectroscopy, but we shall restrict our attention to experiments and instruments at steady state sources (research reactors). Furthermore we shall only be concerned with direct geometry inelastic scattering instruments. The terms "direct geometry" and "inelastic scattering" will first be explained.

In a diffraction experiment no attempt is made to measure the change in energy of the neutrons when they are scattered. Structural information is obtained, but there is no way to extract the temporal (time-dependent) behavior of a system, i.e. dynamical information, from the results of diffraction experiments. To do so requires that the scattered intensity be measured as a function of the neutron's change in energy on scattering, i.e. the neutron's energy transfer. Elastic scattering is scattering with no energy transfer and inelastic scattering is scattering with nonzero energy transfer. An instrument that is used to measure intensity as a function of energy transfer is called an inelastic scattering instrument.

Neutron scattering instruments consist of a "primary spectrometer", which prepares the beam that strikes the sample, and a "secondary spectrometer", which manipulates and detects neutrons scattered by the sample. There are two basic types of time-of-flight inelastic scattering instruments. In direct geometry instruments the incident beam is not only pulsed but monochromated. In other words a single energy or wavelength is selected from the polychromatic beam that emerges from the reactor. The scattered neutron intensity is then measured as a function of the scattering angle, and of the time that each neutron is detected. In "inverted geometry" instruments the pulsed beam at the sample contains neutrons with many different energies, but the only neutrons that are detected are those with a specific scattered energy. Fig.1 shows schematic representations of direct and inverted geometry instruments, with corresponding distance-time diagrams.

\section{General Remarks}

\section{I.1. Definitions}

Our notation is the same as the notation used in the lecture on powder diffraction. We repeat it for completeness.

A neutron's energy $\mathrm{E}$, wave vector $\mathbf{k}$, velocity $\mathrm{v}$, and wavelength $\lambda$ are related as follows:

$$
|\mathbf{k}|=\frac{2 \pi}{\lambda} ; \quad \lambda=\frac{\mathrm{h}}{\mathrm{mv}} ; \quad \mathrm{E}=\frac{1}{2} \mathrm{mv}^{2}=\frac{\mathrm{h}^{2}}{2 \mathrm{~m} \lambda^{2}},
$$


where $\mathrm{h}$ is Planck's constant and $\mathrm{m}$ is the mass of the neutron. The most commonly used unit of neutron energy is the milli-electron volt $(\mathrm{meV}) ; 1 \mathrm{meV} \approx 8.1 \mathrm{~cm}^{-1} \approx 11.6 \mathrm{~K} \approx$ $0.24 \times 10^{12} \mathrm{~Hz} \approx 1.6 \times 10^{-15} \mathrm{erg}$. A neutron with a wavelength of $2 \AA(0.2 \mathrm{~nm})$ has an energy of $\sim 20.5 \mathrm{meV}$, its wave vector is $\sim 3.1 \AA^{-1}$, and it travels $\sim 2 \mathrm{~mm}$ in $1 \mu \mathrm{s}$, i.e. $\sim 2 \mathrm{~m}$ in $1 \mathrm{~ms}$.

In a scattering experiment the incident and final neutron energies are $E_{i}$ and $E_{f}$ respectively and the corresponding wave vectors are $\mathbf{k}_{\mathrm{i}}$ and $\mathbf{k}_{\mathrm{f}}$ (fig. 2 ). The energy transfer $\hbar \omega=E_{i}-E_{f}$ is the energy transferred to the sample by the neutron when it is scattered. Thus $\hbar \omega$ is positive if the neutron loses energy when it is scattered; this is called "downscattering". Conversely $\hbar \omega<0$ if the neutron gains energy, i.e. if it is "upscattered". The wave vector transfer $\mathbf{Q}=\mathbf{k}_{\mathrm{i}}-\mathbf{k}_{\mathrm{f}}$. The cosine rule gives $\mathrm{Q}^{2}=\mathrm{k}_{\mathrm{i}}^{2}+\mathrm{k}_{\mathrm{f}}^{2}-2 \mathrm{k}_{\mathrm{i}} \mathrm{k}_{\mathrm{f}} \cos (2 \theta)$, where $2 \theta$ is the scattering angle. For elastic scattering, $E_{i}=E_{f}, \hbar \omega=0, k_{i}=k_{f}$, and $Q=2 k_{i} \sin \theta=4 \pi \sin \theta / \lambda_{i}$ where $\lambda_{i}$ is the incident wavelength.

\section{I.2. The Time-of-Flight Method}

The primary section of a direct geometry time-of-flight spectrometer, i.e. the part that precedes the sample, includes devices that create a pulsed, monochromated beam of neutrons at the sample position. This can be achieved in various ways. For the moment we shall assume that independent devices pulse and monochromate the beam, and we shall consider a single pulse. Knowing the time that the neutrons were pulsed, the velocity of the monochromated neutrons, and the distance from the pulsing device to the sample, we can calculate the time that neutrons reach the sample. Then, knowing the time that a neutron is detected, and the distance from the sample to the detector, we can calculate the neutron's time-of-flight from sample to detector and hence its scattered velocity. We can therefore calculate its energy transfer and, since the scattering angle is also known, its wave vector transfer. Thus we can map the experimental variables $2 \theta$ and t into the variables $\hbar \omega$ and $\mathbf{Q}$ that characterize the interaction of the neutron with the sample.

\section{I.3. Examples of Instruments}

There are two common methods of producing the pulsed, monochromated beam at the sample position in a direct geometry time-of-flight spectrometer. The time-of-flight instruments at the NIST Center for Neutron Research (NCNR) illustrate these methods (Copley and Udovic, 1993). In the Fermi Chopper Spectrometer (FCS) (fig. 3) the beam from the reactor is monochromated using a double crystal device that produces a single wavelength beam parallel to the reactor beam. It is then pulsed using a "Fermi chopper", which is a device that spins about an axis normal to the axis of the beam. As its name implies, the Disk Chopper Spectrometer (DCS) uses "disk choppers", which are choppers that spin about an axis parallel to the beam direction. The simplest conceivable instrument of this type has two disks rotating at the same speed (fig. 4). The first disk pulses the beam and the second disk only transmits the neutrons whose velocities are such that they arrive at times when the slot in the disk is open. In actual practice additional choppers are required. For example at least one additional disk is needed 
because a system of two disks transmits more than one wavelength, depending on the number of times that the disks have rotated while the neutrons were travelling between the disks. This is illustrated in fig. 5. For various reasons, which are beyond the scope of this lecture, the DCS instrument at the NCNR actually has seven disks.

The rest of the instrument is essentially the same for both types of spectrometers. The principal components include a sample chamber, a flight chamber, and an array of detectors, mounted at one or more well-defined distances from the sample.

\subsection{Theory}

In the lecture about powder diffraction we stated that the scattered intensity in a neutron diffraction experiment on a single crystal is proportional to

$$
S(\mathbf{Q})=\frac{1}{N}\left|\sum_{i=1}^{N} b_{i} \exp \left(i \mathbf{Q} \cdot \mathbf{r}_{i}\right)\right|^{2},
$$

where the sum is over all $\mathrm{N}$ atoms in the crystal, $\mathbf{r}_{\mathrm{i}}$ is the position of atom $\mathrm{i}$, and $\mathrm{b}_{\mathrm{i}}$ is the scattering length of atom $i$. One of the many simplifying assumptions was that all unit cells were identical. This implied that the atoms did not move, so that the scattering was elastic. In order to understand inelastic scattering we must clearly remove the assumption that the atoms do not move!

In a time-of-flight experiment the measured quantity is an intensity which is a function of the scattering angle $2 \theta$ and the time-of-flight $t$. This intensity can be transformed into a more fundamental quantity, variously called the "scattering function", "scattering law" or "dynamic structure factor". Written as $S(\mathbf{Q}, \omega)$, it is a function of the neutron's wave vector transfer $\mathbf{Q}$ and its energy transfer $\hbar \omega$. Equivalently, and more importantly, it is a function of the momentum, $\hbar \mathbf{Q}$, and the energy, $\hbar \omega$, transferred to the system being studied. The details of the transformation from the measured intensity to the scattering function are beyond the scope of these notes, but the point to be remembered is that $S(\mathbf{Q}, \omega)$ describes the dynamical behavior of the system. It is the Fourier transform of another quantity that plays a prominent role in discussions of inelastic scattering, the socalled "intermediate scattering function" $I(Q, t)$. In other words $S(Q, \omega)$ is the power spectrum of $I(Q, t)$. The intermediate function may be written as

$$
I(Q, t)=\frac{1}{N} \sum_{i, j} b_{i} b_{j} \exp \left(i Q \cdot\left[r_{i}(0)-r_{j}(t)\right]\right),
$$

where $\mathbf{r}_{j}(t)$ is the position of atom $j$ at time $t$; notice that $I(Q, 0)=S(Q)$. Furthermore $I(Q, t)$ is independent of $t$ if the atoms are assumed to be stationary, in which case its Fourier transform $S(Q, \omega)$ is only nonzero when $\omega=0$, so that the scattering is purely elastic as expected. For a detailed discussion of the theory of thermal neutron scattering, see Squires (1978) or Lovesey (1987).

Equation (3) must be modified if there is isotopic disorder and/or nuclear spin disorder. In this case $\mathbf{I}(\mathbf{Q}, \mathrm{t})$ is the sum of a coherent function

$$
I_{C}(\mathbf{Q}, t)=\frac{1}{N} \sum_{i, j}\left\langle b_{i}\right\rangle\left\langle b_{j}\right\rangle \exp \left(i \mathbf{Q} \cdot\left[r_{i}(0)-r_{j}(t)\right]\right)
$$


and an incoherent function

$$
I_{I}(\mathbf{Q}, t)=\frac{1}{N} \sum_{i}\left[\left\langle b_{i}^{2}\right\rangle-\left\langle b_{i}\right\rangle^{2}\right] \exp \left(i Q \cdot\left[\mathbf{r}_{i}(0)-\mathbf{r}_{i}(t)\right]\right)
$$

The incoherent scattering is a sum of contributions from individual atoms whereas the coherent scattering reflects correlations between all pairs of atoms.

The incoherent scattering, as manifested in a powder diffraction experiment (i.e. an experiment with no analysis of energy transfer), is independent of Q. As such it contains no useful information. On the other hand the inelastic incoherent scattering is of considerable interest. For example, the space Fourier transform of $\mathrm{I}_{\mathbf{I}}(\mathbf{Q}, \mathrm{t})$ is a spatial correlation function which measures the probability that an atom moves the vector distance $\mathbf{r}$ in time $t$. Thus the inelastic incoherent scattering function yields information about self-diffusion.

\section{I.5. Neutron Kinematics}

In a time-of-flight instrument the energy of each scattered neutron is easily related to its time-of-flight from the sample to the detector. The scattered energies of the detected neutrons vary, at least in principle, from $\infty$ to 0 . The corresponding range of energy transfers $\hbar \omega=E_{i}-E_{f}$ is from $-\infty$ to $E_{i}$. For any given value of $\hbar \omega$, i.e. for any given value of $E_{f}$, the range of $Q$ is limited. The minimum value of $Q$ is $\left|k_{i}-k_{f}\right|$, corresponding to scattering in the forward direction $\left(2 \theta=0^{\circ}\right)$. Similarly the maximum value of $Q$, equal to $\mathrm{k}_{\mathrm{i}}+\mathrm{k}_{\mathrm{f}}$, occurs when $2 \theta=180^{\circ}$. In any experiment the range of $\mathrm{Q}$ is somewhat more restricted since scattering angles of $0^{\circ}$ and $180^{\circ}$ cannot be realized. A typical example of $Q$ range plotted as a function of $\hbar \omega$, for various choices of incident wavelength, is shown in fig. 6. We see that the kinematically allowed region, which is the accessible region in $(\mathbf{Q}, \omega)$ space, becomes larger as the incident wavelength is reduced.

\section{I.6. Resolution and Intensity Considerations}

An important advantage of a time-of-flight instrument such as the NCNR DCS is its versatility. It can be operated in various ways, representing different tradeoffs between resolution and intensity. The incident wavelength is easily varied by changing the phasing of the choppers. As the wavelength is increased the resolution of the instrument improves, but the $\mathrm{Q}$ range becomes more restricted and the range of accessible energy transfers in downscattering (which is generally more intense, especially at low temperatures) is severely reduced (fig. 6). Furthermore the fact that the neutrons are travelling more slowly means that it becomes increasingly likely that "frame overlap" will be a problem. (Frame overlap is considered to occur when the slowest neutrons associated with a given pulse arrive at the detector later than the fastest neutrons associated with the following pulse, leading to ambiguities in interpretation of the data, as illustrated in fig. 7.)

For these reasons there is much to be said for trying to obtain good resolution without having to resort to the use of unnecessarily long wavelength neutrons. This is achieved at the DCS through the use of multiple slots in some of the choppers, effectively permitting 
the use of beams of different widths and burst times. As always, the price paid for the improved resolution is a significant loss in intensity.

\section{I.7. Types of Scattering}

Setting to one side the neutron spin echo technique, reactor-based neutron scattering instruments for inelastic scattering experiments may be broadly classified as crystal spectrometers or time-of-flight spectrometers. Triple-axis crystal spectrometers are particularly suited to studies of excitations in single crystals, in which case the scattered intensity is confined to relatively small volumes in $(\mathbf{Q}, \omega)$ space. Typically a triple-axis instrument measures count-rates with high efficiency but it only looks at one point at a time in $(\mathbf{Q}, \omega)$ space. On the other hand time-of-flight instruments can be used to examine large regions in $(\mathbf{Q}, \omega)$ space because data are simultaneously collected in many detectors and over a broad range of energy transfers. The disadvantage of the technique is that count-rates in any given time channel and detector channel are much lower because the incident beam has been pulsed and is therefore relatively weak. Hybrid time-of-flight instruments, i.e. those that use monochromator crystals, are somewhat less versatile than those that do not use crystals, in that the range of available incident wavelengths is more restricted. The latter comparison is only significant if the instruments are located at a source of cold neutrons.

The scattering that is studied with a time-of-flight spectrometer is by definition inelastic since scattered intensity is measured as a function of neutron energy transfer. Nevertheless the term "quasielastic" is frequently used to describe a particular type of inelastic scattering that is (mostly) studied using time-of-flight techniques. Quasielastic scattering (Bée, 1988) is scattering that is centered at zero energy transfer and has intrinsic width. On the other hand, elastic scattering is also centered at zero energy transfer, but has no intrinsic width. Quasielastic scattering, as observed, is scattering that is broader than the instrumental resolution whereas elastic scattering has the width of the instrumental resolution (fig. 8). The phrase "inelastic scattering", in the present context, is generally reserved for scattering that is centered at nonzero energy transfers, as illustrated in fig. 8 .

The energy dependence of the scattering intensity tells us about the dynamical behavior of the system that is being studied. For example a narrow inelastic peak, with a welldefined energy $\hbar \omega$, implies some sort of periodic motion such as torsion or bondbending, with a well defined period $1 / \omega$. The broader the peak, the shorter the lifetime of the motion. On the other hand quasielastic scattering measures diffusional motion and the width of the scattering is inversely related to the time scale of the motion. Slow diffusional processes give rise to narrow quasielastic peaks, which require the use of an instrument with very good energy resolution.

One of the advantages of neutron scattering as a spectroscopic tool is that spatial information can be extracted from the results of a measurement. For example the $Q$ dependence of the quasielastic scattering can be used to distinguish among competing models of jump diffusional behavior, such as jumps among different types of lattice sites. 
The following paragraphs contain additional examples of information extracted from the $\mathbf{Q}$ dependence of the scattering function.

\section{Applications}

We shall describe several applications of time-of-flight spectroscopy.

\section{II.1. Refrigerants in Zeolites}

Hydrofluorocarbons (HFCs) are gradually replacing chlorofluorocarbons (CFCs) in refrigeration and air-conditioning applications as ozone-depleting CFCs are phased out in accordance with international agreements. Zeolites such as $\mathrm{Na}-\mathrm{X}\left(\mathrm{Na}_{86} \mathrm{Al}_{86} \mathrm{Si}_{106} \mathrm{O}_{384}\right)$ can be used to separate HFCs because they preferentially adsorb certain species. For example, $\mathrm{HFC} 134\left(\mathrm{HF}_{2} \mathrm{C}-\mathrm{CF}_{2} \mathrm{H}\right)$ is produced alongside the prototypical refrigerant $\mathrm{HFC134a}$ $\left(\mathrm{F}_{3} \mathrm{C}-\mathrm{CFH}_{2}\right)$, and $\mathrm{Na}-\mathrm{X}$ could possibly be used to separate the isomers. In order to understand the interactions responsible for such processes it is appropriate to investigate the structure and dynamics of these types of systems. With regard to structure, the $\sim 20 \%$ difference between the neutron scattering lengths of $\mathrm{Al}$ and $\mathrm{Si}$, as compared with the $\sim 8 \%$ difference in the $\mathrm{x}$-ray atomic scattering factors, has enabled crystallographers to conduct neutron diffraction experiments that unambiguously determine the positions of the $\mathrm{Al}$ and $\mathrm{Si}$ species in Na zeolites and there are plans to study the structures of the adsorbed systems.

Inelastic neutron scattering methods have been used to study the vibrational spectra of both HFC134 and HFC134a, bound in Na-X (Udovic et al, 1995). The higher energy excitations were studied by filter-analyzer spectroscopy (Copley et al, 1995) and the lower energy excitations were investigated using the Fermi Chopper Spectrometer at the NCNR. The results of the FCS measurements on saturated materials (containing $\sim 4$ molecules per zeolite supercage), and on unloaded $\mathrm{Na}-\mathrm{X}$, are shown in fig. 9. These results were obtained in neutron energy gain, using $5 \AA(\sim 4 \mathrm{meV})$ incident neutrons. The spectra are remarkably different, in part because of differences in the symmetries of the adsorbed molecules but also because of differences in how the molecules attach to the zeolite. A detailed discussion of the various features is beyond the scope of these lecture notes, but suffice to say that there is evidence that the gas-phase torsional and C-H stretching vibrations are modified when the molecules are adsorbed, and that additional features can be related to whole molecule vibrations and adsorbate-coupled zeolite framework vibrations. Combining the neutron data with the results of other spectroscopies, it is hoped that a detailed picture of the binding forces and guest-host interactions will be forthcoming.

\section{II.2. Curing of Cement}

The mechanisms and kinetics of the reactions that occur in the setting and hardening of Portland cement are at best imperfectly understood. The better they can be understood, the more likely it is that we shall see advances in methods of construction using concrete. Conditions and procedures for mixing and pouring may be better optimized and the results of such operations in a variety of environments may be more predictable. There is also the possibility of improvements in the mechanical properties of the final product. 
Neutron scattering methods have recently been used by Fitzgerald et al (1997) to investigate the most important reaction that occurs when Portland cement is mixed with water, the hydration of tricalcium silicate, $\mathrm{Ca}_{3} \mathrm{SiO}_{5}$, to form an amorphous gel-like material called $\mathrm{CSH}$, together with crystalline calcium hydroxide, $\mathrm{Ca}(\mathrm{OH})_{2}$; it is the $\mathrm{CSH}$ that gives strength to the final product. The overall reaction may be written as

$$
\mathrm{Ca}_{3} \mathrm{SiO}_{5}+(3+\mathrm{y}-\mathrm{x}) \mathrm{H}_{2} \mathrm{O} \Rightarrow(\mathrm{CaO})_{x}\left(\mathrm{SiO}_{2}\right) \cdot\left(\mathrm{H}_{2} \mathrm{O}\right)_{\mathrm{y}}+(3-\mathrm{x}) \mathrm{Ca}(\mathrm{OH})_{2}
$$

where $\mathrm{x}$ and $\mathrm{y}$ are quantities that change over the course of the reaction and vary within the sample. The overall reaction actually consists of three separate reactions and the progress of each of these reactions depends on various factors such as the morphology of the starting material. An important measure of the progress of these reactions is the "free water index" (FWI), which is essentially the amount of free water remaining divided by the total water that was initially in the sample. The FWI can be measured by neutron scattering.

The measurements were performed using the NCNR FCS. The incident wavelength was $4.8 \AA$ and data were collected over a range of scattering angles. The energy resolution of the instrument was $0.146 \mathrm{meV}$ for elastically scattered neutrons. The sample was a $0.5 \mathrm{~mm}$ thick paste of $\mathrm{Ca}_{3} \mathrm{SiO}_{5}$ mixed with distilled water ( $\sim 28 \%$ by weight). (The sample had to be relatively thin, by neutron standards, because hydrogen has an abnormally large incoherent scattering cross section; a thicker sample would introduce data analysis problems because of severe multiple scattering.)

Fig. 10 shows the time evolution of the scattering for a sample cured at $20^{\circ} \mathrm{C}$. At short times the scattering is broad in energy, signifying that virtually all of the water is mobile; the energy width of the scattering is directly proportional to the mobility of the water. At long times the scattering has two components of roughly equal integrated intensity; the narrow distribution corresponds to water that was immobilized when it reacted with $\mathrm{Ca}_{3} \mathrm{SiO}_{5}$ whereas the broad component represents the water that is still free. Results for the FWI, from measurements on samples cured at different temperatures, are shown in fig. 11. In every case there is an initial induction period which lasts from about 30 minutes at $40^{\circ} \mathrm{C}$ to $\sim 16$ hours at $10^{\circ} \mathrm{C}$. This is followed by a period of nucleation and growth on the surfaces of the $\mathrm{Ca}_{3} \mathrm{SiO}_{5}$ grains, during which time $\mathrm{CSH}$ and $\mathrm{Ca}(\mathrm{OH})_{2}$ begin to form. The nucleation and growth process ends at times that vary from $\sim 6$ hours at $40^{\circ} \mathrm{C}$ to $\sim 80$ hours at $10^{\circ} \mathrm{C}$, as indicated by the arrows in fig. 11. Thereafter the rate of reaction slows, becoming diffusionlimited as the reaction proceeds from the surface to the interior of the $\mathrm{Ca}_{3} \mathrm{SiO}_{5}$ grains. Future experiments are planned to determine the rate of formation of $\mathrm{Ca}(\mathrm{OH})_{2}$, by looking at the time dependence of the intensity of a characteristic $\mathrm{Ca}-\mathrm{O}$ stretching mode.

\section{II.3. Hydrogen in Metals}

The motion of hydrogen atoms in metals is relatively easily observed in neutron scattering experiments because of the large incoherent scattering cross section of hydrogen. A good example is a recent study of hydrogen dynamics in rare earth hydrides. The interest in these materials is that as thin films they become optically transparent when the hydrogen/metal ratio approaches three. In an effort to improve our understanding of the role of the hydrogen 
atoms in this phenomenon, Karmonik et al (1997) have performed time-of-flight quasielastic neutron scattering experiments on $\mathrm{YH}_{3}$ and $\mathrm{LaH}_{3-\mathrm{x}}$, with $0<\mathrm{x} \leq 0.5$, using the NCNR FCS. Representative results are shown in fig. 12.

In the case of hexagonal close packed $\mathrm{YH}_{3}$ there seems to be no evidence of scattering other than a line that is purely elastic within the resolution of the experiment. An analysis of earlier neutron powder diffraction data had suggested that there were possible channels for hydrogen motion within the crystal lattice. It is possible that the time scale of this diffusional motion is too long, or perhaps the diffusion distance is too small, for quasielastic scattering to be observable using the FCS. Experiments with much better energy resolution should be able to shed light on this question.

The scattering by face-centered cubic $\mathrm{LaH}_{3-\mathrm{x}}$, with $\mathrm{x}$ believed to be approximately 0.01 , is considerably more interesting and informative. In this case the observed scattering is well fitted by two Lorentzian functions with different widths, plus a background (fig. 12). The behaviors of the widths and intensities of the Lorentzian components, as functions of $\mathrm{Q}$, suggest that two types of motion are going on. The faster motion appears to be some sort of localized rattling within the spacious octahedral cavities of the lattice; the Q dependence of the scattering is consistent with a model in which atoms at octahedral sites move on a sphere of radius $0.23 \AA$. The slower motion can be quantitatively explained as a hopping of hydrogen vacancies among octahedral sites within the lattice, via the fully occupied tetrahedral sites; the hopping distance extracted from the data is close to the known distance between octahedral and tetrahedral sites. These results demonstrate the power of neutron scattering as a way of examining spatial as well as temporal aspects of dynamical processes.

\section{II.4. Battery Anode Materials}

In recent years there has been considerable progress in the development of rechargeable lithium batteries. One class of material that is used for the anodes of such batteries is disordered carbon, and in order to maximize the stored energy per unit mass it is important to determine which types of disordered carbon react reversibly with the largest amount of lithium. It is known that materials prepared by pyrolyzing organic solids at high temperatures in an inert gas atmosphere have surprisingly large Li uptake capacities, of order $900 \mathrm{mAh} / \mathrm{g}$, and that they also contain considerable amounts of hydrogen. For example chemical analyses, including prompt gamma neutron activation analysis (Neumann et al, 1993), showed that a material that we shall call DC650, prepared from an epoxy resin at $650^{\circ} \mathrm{C}$, contained $0.24 \pm 0.01$ hydrogen atoms per carbon atom. It is believed that these types of disordered carbons consist of planar aromatic structures called graphene sheets, terminated with hydrogen atoms at their edges. Calculations predict that lithium not only intercalates into the sheets but also binds to the H-terminated carbon atoms.

Several disordered carbon anode materials have been studied using neutron techniques including time-of-flight diffraction, small angle scattering, filter-analyzer spectroscopy and time-of-flight quasielastic scattering (Zhou et al, 1997). The ultimate goal of this work is to understand the very high capacity of these materials for $\mathrm{Li}$ uptake. Filter-analyzer measurements have recently been performed on DC650, on coronene $\left(\mathrm{C}_{24} \mathrm{H}_{12}\right.$, a planar molecule with graphite hexagons surrounded by $-\mathrm{CH}=$ groups), and on polyethylene, 
$\left(-\mathrm{CH}_{2}-\right)_{\mathrm{n}}$. The results of these experiments (Papanek et al, 1997) strongly support the proposition that single protons attach to the edge carbons in a material such as DC650, affording the possibility that $\mathrm{Li}$ atoms can also bind to these sites. Filter-analyzer results for DC650 at 16K, before and after loading with $\mathrm{Li}$, are shown in fig. 13, and results at lower energy transfer, obtained using the NCNR Fermi Chopper Spectrometer, are shown in fig. 14 (Papanek et al, 1997). The measured $\mathrm{Li} / \mathrm{C}$ ratio in the doped material was slightly less than $1 / 3$, considerably greater than the maximum loading of $\mathrm{Li}$ in graphite, which is $1 / 6$. There is little difference between the spectra for the undoped and doped materials, except at the lowest energy transfers (fig. 14), where the scattering has clearly shifted to higher energies in the doped material. This observation is consistent with the picture that $\mathrm{Li}$ intercalates into the graphitic layers, stiffening the shear modes responsible for the scattering at these energy transfers and hardening the spectral response.

\section{II.5. Carbon-60}

In the lecture on powder diffraction we discussed neutron studies of the crystal and molecular structures of $\mathrm{C}_{60}$. We turn now to a brief description of measurements of the low energy dynamics of $\mathrm{C}_{60}$. The internal modes of the molecule, i.e. the intramolecular dynamics, have been studied using the filter-analyzer technique (Copley et al, 1995) but we shall not discuss this work. Nor shall we discuss single crystal studies of the external translational modes, i.e. the phonon dispersion curves. We shall instead concentrate on the reorientational dynamics of the molecules, both above and below the first order phase transition which occurs at $\mathrm{T}_{c} \approx 260 \mathrm{~K}$. Though the work that we shall describe was performed at NIST using a triple-axis spectrometer, there is no reason that it could not have been performed by time-of-flight, had an appropriate instrument been available at the time.

Above $T_{c}$, neutron diffraction tells us that $\mathrm{C}_{60}$ adopts a face-centered cubic structure with all molecules structurally equivalent, and that to first order the molecules are randomly oriented. On the other hand it tells us nothing about the temporal behavior of the molecules, e.g. whether or not the orientational disorder is static or dynamic. In diffraction experiments the disorder manifests itself as intense diffuse scattering between and below the Bragg peaks, as shown in fig. 15. To investigate the time scale of the disorder we have performed inelastic scattering measurements at several values of $Q$.. These measurements reveal that the scattering from the C60 is quasielastic rather than elastic, as illustrated in fig. 16. (The elastic scattering that is observed, particularly at smaller wave vectors, is believed to be due to residual hydrogen in the sample.) The observed quasielastic scattering is reasonably well described by a simple random walk diffusional model in which it is assumed that the molecules tumble through all possible orientations and that there are no correlations between the orientations of different molecules. This model clearly oversimplifies the situation but, to the extent that it is a very good first approximation, we find that the rotational diffusion constant, which determines the time scale of the reorientational motion, is $1.4 \pm 0.4 \times 10^{-10} \mathrm{~s}^{-1}$, implying that $\mathrm{C}_{60}$ molecules in the solid state reorient somewhat faster than they do in solution.

There is a dramatic change in the low energy dynamics of $\mathrm{C}_{60}$ as it is cooled through the phase transition temperature (fig. 17). The quasielastic scattering above $T_{c}$ changes to 
inelastic scattering below $T_{c}$, together with a relatively strong elastic peak which is attributed to the tails of nearby resolution-broadened Bragg peaks. (One of the challenges in these experiments was that it was necessary to work at relatively high $\mathrm{Q}$ because of the very high symmetry of the molecule, and yet good $Q$ resolution was needed in order to minimize Bragg peak contamination of the low energy inelastic scattering; in this regard we were not completely successful.) From the $Q$ dependence of the intensities of the inelastic peaks at temperatures below $T_{c}$, we conclude that the peaks are due to librational motions of molecules about their equilibrium orientations. Given the measured librational frequency and the known moment of inertia of the $\mathrm{C}_{60}$ molecule, and assuming that the orientational potential can be treated as a sinusoidal function of the angular displacement of the molecule, we can determine the height of the potential barrier if we also know the angular separation between potential minima. The results of time-of-flight powder diffraction measurements on $\mathrm{C}_{60}$, described in the powder diffraction lecture, can be explained by a model that allows each molecule to adopt two types of orientations ("pentagon-facing" and "hexagon-facing"), and from the temperature dependence of the fitted probability that a molecule adopts the pentagon-facing orientation the free energy difference between the two types of orientations is $\approx 11 \mathrm{meV}$. Since this free energy difference is small, and since the angular separation between neighboring pentagonfacing and hexagon-facing orientations (which is $\approx 42^{\circ}$ ) is considerably less than the angular separation between nearest neighbor pentagon-facing orientations, we use this angle to determine the height of the barrier. The result is $\approx 245 \mathrm{meV}$, which is in good agreement with the results of a number of experimental measurements over a wide range of experimental time scales (Neumann et al, 1993).

\section{II.6. Cubane}

As a final example we shall discuss some recent work on solid cubane, $\mathrm{C}_{8} \mathrm{H}_{8}$. First synthesized in 1964, cubane is a molecule with carbon atoms at the corners of a cube, with a hydrogen atom attached to each carbon. The C-C-C bond angle is $90^{\circ}$ rather than the $109.5^{\circ}$ that is customarily found in most hydrocarbon molecules. Thus the bonds are highly strained and cubane is regarded as an ideal candidate for novel high energy density materials such as explosives and propellants. Replacing the hydrogen atoms with $\mathrm{NO}_{2}$ groups would produce an even better material for such applications. Other types of replacements could possibly result in pharmaceutical derivatives to combat diseases such as AIDS and cancer. A group at NIST has recently undertaken a comprehensive investigation into the structural and dynamical properties of this remarkable material (Yildirim et al, 1997a). The structure was studied using x-ray diffraction; neutrons could not have been used because the incoherent scattering from the hydrogen would have swamped the diffraction pattern. On the other hand neutron time-of-flight spectroscopy was used to investigate the low energy dynamics, taking full advantage of the large incoherent scattering cross section of hydrogen.

Measurements using the NCNR FCS have been analyzed to obtain the intermolecular component of the vibrational density of states (VDOS) of cubane at 100 and $300 \mathrm{~K}$, shown in fig. 18 (Gehring et al, 1995). The intermolecular vibrations are the coupled whole body motions of the molecule. In the limit that intermolecular interactions become very weak, these vibrations become the three acoustic translational (oscillatory) modes 
and the three librational (rocking) modes of an isolated molecule. At low frequencies, up to about $60 \mathrm{~cm}^{-1}$, the VDOS curves at the two temperatures are similar (fig. 18), but at higher frequencies there are marked differences. Three distinct peaks are observed at $100 \mathrm{~K}$ whereas at $300 \mathrm{~K}$ the VDOS is almost flat from $\sim 60$ to $\sim 100 \mathrm{~cm}^{-1}$. The explanation, which is nicely confirmed by theoretical calculations, is that the librational modes occur at higher frequencies than the translational modes, and that with increasing temperature the librational modes soften (i.e. their frequencies decrease) to a much greater extent than the translational modes. Such a softening is consistent with the experimental observation that an order-disorder transition to an orientationally-disordered phase occurs at 394K. The peaks in the $100 \mathrm{~K}$ VDOS at 94 and $114 \mathrm{~cm}^{-1}$ are believed to correspond to librational modes, whereas transverse acoustic translational modes are thought to be responsible for the peak at $78 \mathrm{~cm}^{-1}$.

At temperatures below the $394 \mathrm{~K}$ order-disorder phase transition temperature, cubane is rhombohedral with one molecule per unit cell. Surprisingly the high temperature phase (above $394 \mathrm{~K}$ ) is also rhombohedral (rather than cubic), again with one molecule per unit cell. The x-ray diffraction data rule out spherical disorder (such as exists in room temperature $\mathrm{C}_{60}$ ), as well as uniaxial rotational disorder about the rhombohedral axis. Quasielastic neutron scattering measurements at $385 \mathrm{~K}$ and $400 \mathrm{~K}$ (Yildirim et al, 1997b) have been analyzed to obtain the so-called elastic incoherent structure factor (EISF) as a function of Q; the EISF is simply the ratio of the intensity in the elastic peak to the total integrated intensity. The results of this analysis are shown in fig. 19, together with the predictions of various jump-diffusion models. Isotropic rotation is clearly ruled out, as are 4-site $\left(90^{\circ}\right)$ jumps about four-fold axes and, perhaps less obviously, 2-site $\left(180^{\circ}\right)$ jumps about two-fold axes. On the other hand the $385 \mathrm{~K}$ measurements are in good agreement with models based on 3-site $\left(120^{\circ}\right)$ jumps about three-fold axes and on 2-site $\left(180^{\circ}\right)$ jumps about four-fold axes. Since model calculations predict a much smaller barrier to rotation about a four-fold axis, this is believed to be the most likely mechanism for the observed jump diffusion. Details of what is happening in the disordered phase, at $400 \mathrm{~K}$, are less clear cut. It may be that several types of jumps are going on at the same time, together with large amplitude anharmonic oscillations about minima in the potential energy of the system, but definitive statements await the development of appropriate theoretical models.

\section{Acknowledgments}

I am grateful to my colleagues at NIST, especially Stephen Fitzgerald, Peter Gehring, Bill Kamitakahara, Christof Karmonik, Nick Maliszewskyj, Dan Neumann, Peter Papanek, Terry Udovic and Taner Yildirim, for useful discussions, for the use of figures, and for the use of unpublished results. My thanks too to Mike Crawford, of E. I. du Pont de Nemours and Company, for helpful advice. 
$\underline{\text { References }}$

M. Bée (1988), "Quasielastic Neutron Scattering" (Adam Hilger, Bristol and Philadelphia).

J.R.D. Copley and T.J. Udovic (1993), J. Res. NIST $\underline{98}, 71$.

J.R.D. Copley, D.A. Neumann and W.A. Kamitakahara (1995), Can. J. Phys. $\underline{73}, 763$.

S.A. Fitzgerald, D.A. Neumann, J.J. Rush, D.P. Bentz and R.A. Livingston (1997), Chem. Mater. (submitted).

P.M. Gehring, D.A. Neumann, W.A. Kamitakahara, J.J. Rush, P.E. Eaton and D.P. VanMeurs (1995), J. Phys. Chem. 99, 4429.

Ch. Karmonik, T.J. Udovic and J.J. Rush (1997), private communication.

S.W. Lovesey (1987), "Theory of Neutron Scattering from Condensed Matter" (2 volumes) (Oxford University Press, Oxford).

D.A. Neumann, J.R.D. Copley, D. Reznik, W.A. Kamitakahara, J.J. Rush, R.L. Paul and R.M. Lindstrom (1993), J. Phys. Chem. Solids $\underline{54}, 1699$.

P. Papanek, W.A. Kamitakahara, O. Zhou and J.E. Fischer (1997), private communication.

G.L. Squires (1978), "Thermal Neutron Scattering" (Cambridge University Press, Cambridge).

T.J. Udovic, J.M. Nicol, R.R. Cavanagh, J.J. Rush, M.K. Crawford, C.P. Grey and D.R. Corbin (1995), Mat. Res. Soc. Symp. Proc. $\underline{376}, 751$.

T. Yildirim, P.M. Gehring, D.A. Neumann, P.E. Eaton and T. Emrick (1997a), Carbon (in press).

T. Yildirim, P.M. Gehring, D.A. Neumann, P.E. Eaton and T. Emrick (1997b), private communication.

O. Zhou, P. Papanek, R. Lee, J.E. Fischer and W.A. Kamitakahara (1997), J. Electrochem. Soc. 144, 1744. 


\section{Figure Captions}

Fig. 1. Schematic examples of direct and inverted geometry instruments. In the corresponding distance-time diagrams, " $\mathrm{d}$ " denotes distance and " $\mathrm{t}$ " denotes time; $\mathrm{P}, \mathrm{S}$ and $\mathrm{D}$ denote the pulser, the sample and the detector respectively, and $\mathrm{M}$ and A denote the monochromator and the analyzer respectively. These devices only reflect a single wavelength.

Fig. 2. A scattering event in real space (a), and the corresponding scattering triangle (b). The symbols are defined in the text.

Fig. 3. A Fermi chopper spectrometer. The Fermi Chopper Spectrometer (FCS) at the NIST Center for Neutron Research (NCNR) is configured as shown in this schematic. Neutrons travel through the channels of the Fermi chopper when they are aligned with the beam. The channels are curved so that neutrons within a range of speeds are transmitted. The mid-point of the range is proportional to the rotational speed of the chopper.

Fig. 4. A schematic of a disk chopper spectrometer. The first chopper pulses the beam and the second chopper selects neutrons that arrive at times when the chopper slot is aligned with the neutron beam. Neutrons are transmitted by a disk chopper when the slot in the chopper is in line with the neutron beam.

Fig. 5. A distance-time diagram showing a pulsing chopper (PC) and a monochromating chopper $(\mathrm{MC})$ at a distance $\mathrm{D}$ downstream. Consider the pulse of neutrons created at time $t_{p}$. In the absence of any other choppers the MC transmits neutrons that originated with this pulse and arrived at the $\mathrm{MC}$ at times $\mathrm{t}_{3}, \mathrm{t}_{-2}, \ldots$, but the only neutrons from this pulse that are wanted are those that arrive at the $\mathrm{MC}$ at time $\mathrm{t}_{0}$. To eliminate the unwanted neutrons a removal chopper ( $\mathrm{RC}$ ) is placed between the $\mathrm{PC}$ and $\mathrm{MC}$, and phased to transmit the desired neutrons. Correct positioning of the RC is critical to the success of this technique.

Fig. 6. Kinematically allowed regions in $(\mathbf{Q}, \omega)$ space for neutrons with wavelengths of 2 , 4 and $8 \AA$. It is assumed that detectors are located at angles $2 \theta$ between $10^{\circ}$ and $140^{\circ}$. Plots of $\mathbf{Q}$ versus $\omega$ for $2 \theta=10^{\circ}$ and $140^{\circ}$ are shown as light and heavy lines respectively. For a given wavelength the kinematically allowed region lies between these two lines. The maximum value of $\hbar \omega$ occurs when incident neutrons lose all their energy.

Fig. 7. A distance-time diagram illustrating the problem of frame overlap. It is assumed that neutrons within a narrow range of wavelengths arrive at the sample position at regular intervals. Neutrons that arrive at the detector at time $t_{D}$ could originate with more than one of the pulses at the sample if the pulses are too close together. To alleviate this ambiguity it is generally necessary to add a slow chopper, whose period is a multiple of the period of the other choppers.

Fig. 8. A schematic representation of the differences between elastic, quasielastic and inelastic scattering. Elastic and quasielastic scattering are both centered at zero energy 
transfer. The elastic scattering has the width of the instrumental resolution. The quasielastic scattering is somewhat broader. Inelastic scattering is centered at nonzero energy transfers. Inelastic peaks are shown in energy loss and energy gain.

Fig. 9. Inelastic neutron scattering spectra obtained using the NCNR Fermi Chopper Spectrometer for two different HFCs in Na-X zeolite. Results for the unloaded $\mathrm{Na}-\mathrm{X}$ are also shown. The sample temperature was $200 \mathrm{~K}$ for each of the measurements.

Fig. 10. Quasielastic spectra for a sample of $\mathrm{Ca}_{3} \mathrm{SiO}_{5}$ cured at $20^{\circ} \mathrm{C}$. Each curve represents intensity accumulated for 30 minutes in several detectors, plotted as a function of energy transfer.

Fig. 11. The time evolution of the free water index for $\mathrm{Ca}_{3} \mathrm{SiO}_{5}$ samples cured at 10, 20, 30 and $40^{\circ} \mathrm{C}$. Note the logarithmic abscissa scale. Changes in slope, indicated by arrows, signal a switch from the nucleation and growth regime to a diffusion-limited type of behavior.

Fig. 12. Quasielastic neutron scattering spectra for $\mathrm{YH}_{3}$ and $\mathrm{LaH}_{3-\mathrm{x}}(\mathrm{x} \approx 0.01)$, measured at high temperatures using $4.8 \AA$ incident neutrons. Differences between theory and experiment are shown at the top and bottom of the figure for the $\mathrm{Y}$ and La compounds respectively. The $\mathrm{YH}_{3}$ data comprise the resolution function (determined from a low temperature measurement) and a linear background. The $\mathrm{LaH}_{3-\mathrm{x}}$ results are fitted to two resolution-broadened Lorentzian functions (also shown) plus a linear background.

Fig. 13. Filter-analyzer spectra for doped and undoped DC650, measured at $16 \mathrm{~K}$. The spectrum for the doped material has been shifted upward for clarity. The abscissa represents energy transfer (neutron energy loss).

Fig. 14. Time-of-flight spectra for pristine DC650 and lithium-doped DC650, measured at $300 \mathrm{~K}$. The lower plot shows the same data on an expanded energy scale. The abscissa represents energy transfer (neutron energy gain).

Fig. 15. The room temperature diffraction pattern of $\mathrm{C}_{60}$. The experimental data are shown as points, and a model calculation is represented by the solid line. Some of the peaks are labelled with the quantity $\mathrm{h}^{2}+\mathrm{k}^{2}+\ell^{2}$, where $\mathrm{h}, \mathrm{k}$ and $\ell$ are Miller indices thath characterize the reflection. The peaks at $\sim 2.7 \AA^{-1}$ and $\sim 3.1 \AA^{-1}$ are due to the aluminum sample container.

Fig. 16. Quasielastic scattering spectra for $\mathrm{C}_{60}$ at $260 \mathrm{~K}$. The lines represent fits of a Lorentzian function convoluted with a Gaussian resolution function. In each case the width of the Gaussian is represented by a solid horizontal bar. The excess elastic intensity, which is particularly apparent at the smaller Q, is believed to be due to hydrogen in the sample.

Fig. 17. Inelastic scattering spectra for $\mathrm{C}_{60}$ above and below the phase transition. The spectra were measured at $\mathrm{Q}=5.65 \AA^{-1}$, at the temperatures indicated. 
Fig. 18. The vibrational density of states of cubane, $\mathrm{C}_{8} \mathrm{H}_{8}$, at $100 \mathrm{~K}$ and $300 \mathrm{~K}$. To convert the energy scale from $\mathrm{cm}^{-1}$ to $\mathrm{meV}$, divide by $\sim 8.1$.

Fig. 19. The elastic incoherent structure factor (EISF) of solid cubane, $\mathrm{C}_{8} \mathrm{H}_{8}$. Theoretical results for several models are shown, along with the experimental data at $385 \mathrm{~K}$ (below the phase transition) and at $400 \mathrm{~K}$ (above the phase transition). The cubane molecule, and various jumps that rotate the molecule into itself, are also illustrated. 


\section{Types of TOF spectrometer}

DIRECT GEOMETRY

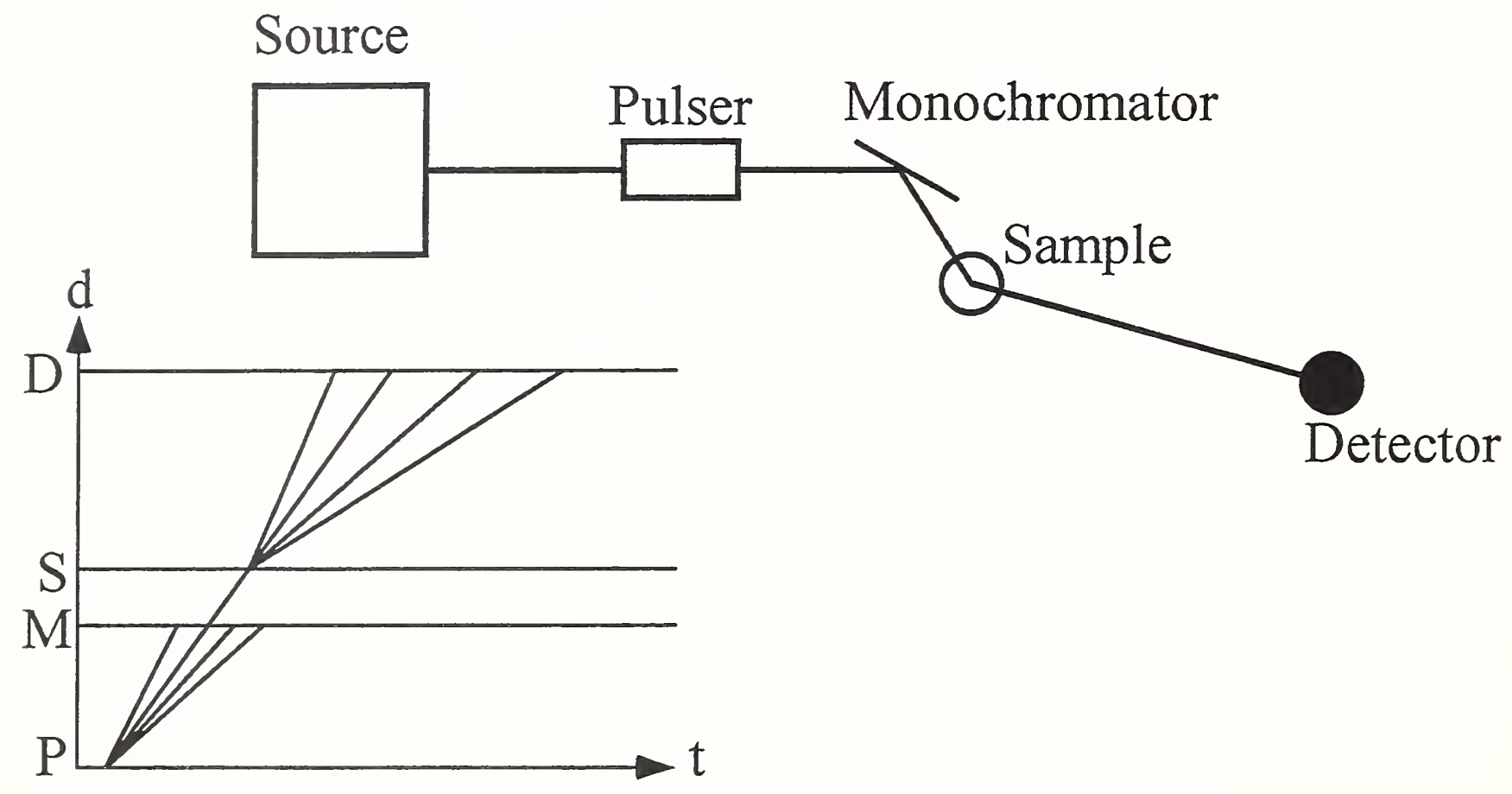

INVERTED GEOMETRY

Source
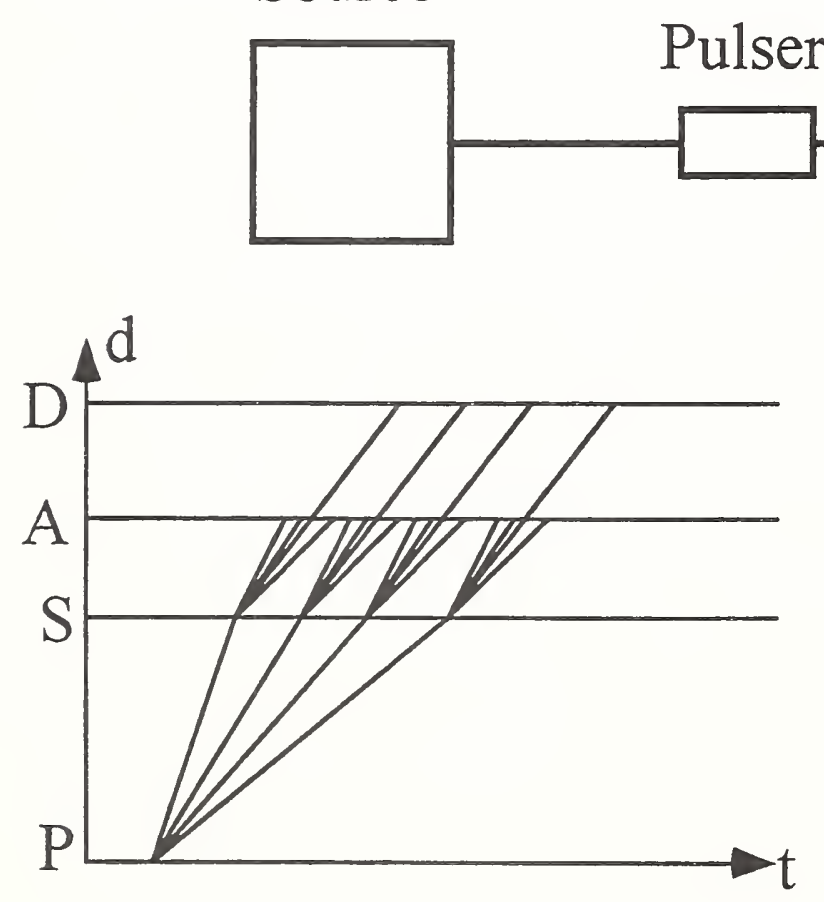

Sample

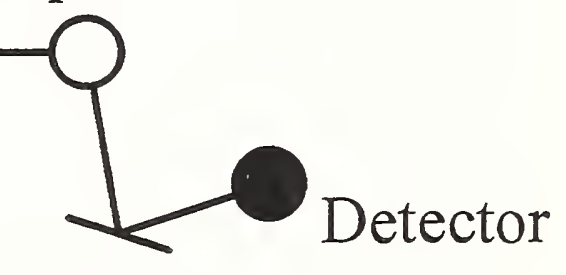

Analyzer

Fig.1 


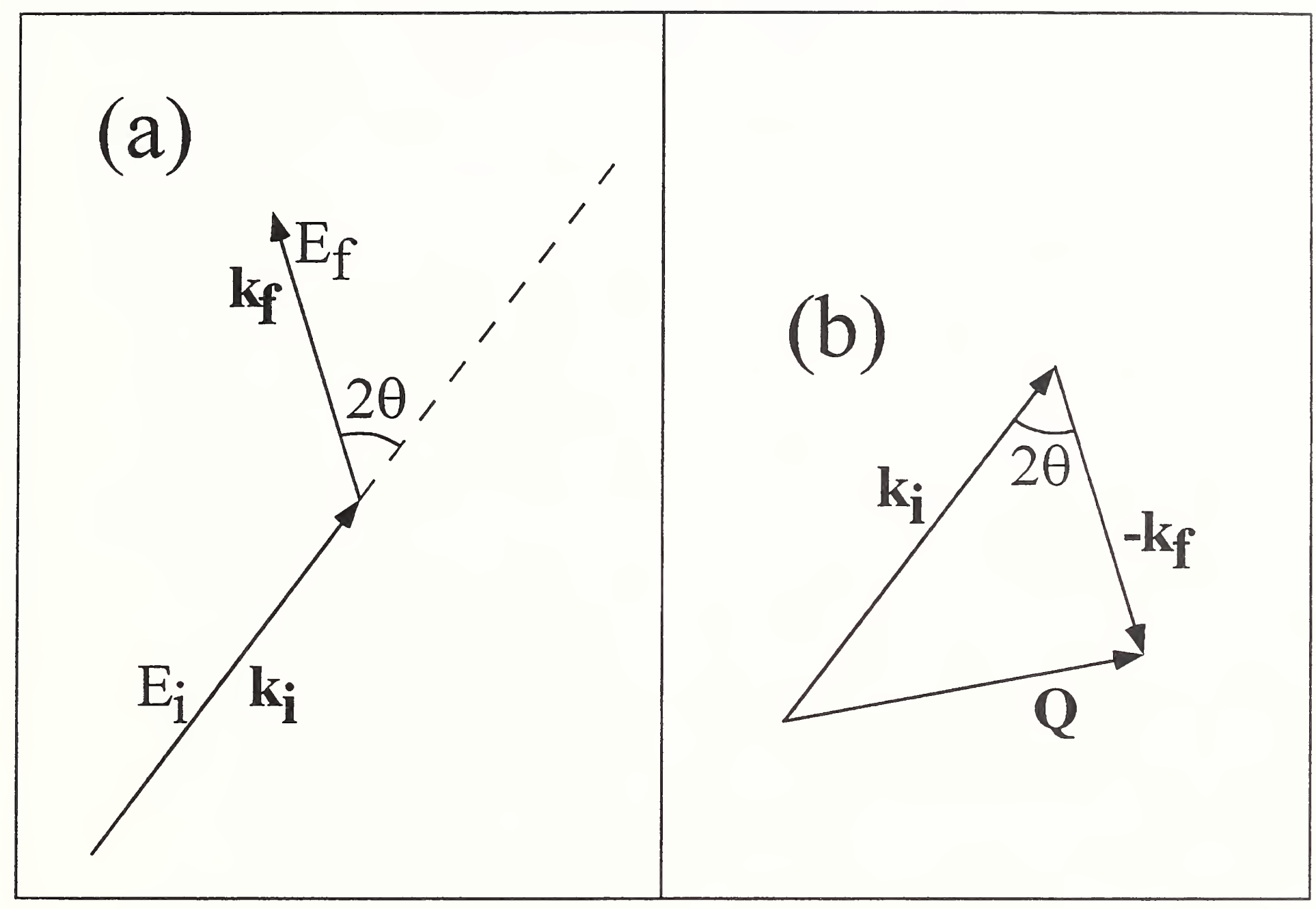

$$
\begin{gathered}
\hbar \omega=E_{i}-E_{f} \\
Q=k_{i}-\mathbf{k}_{\mathbf{f}}
\end{gathered}
$$

Fig.2 


\section{Fermi Chopper Spectrometer}

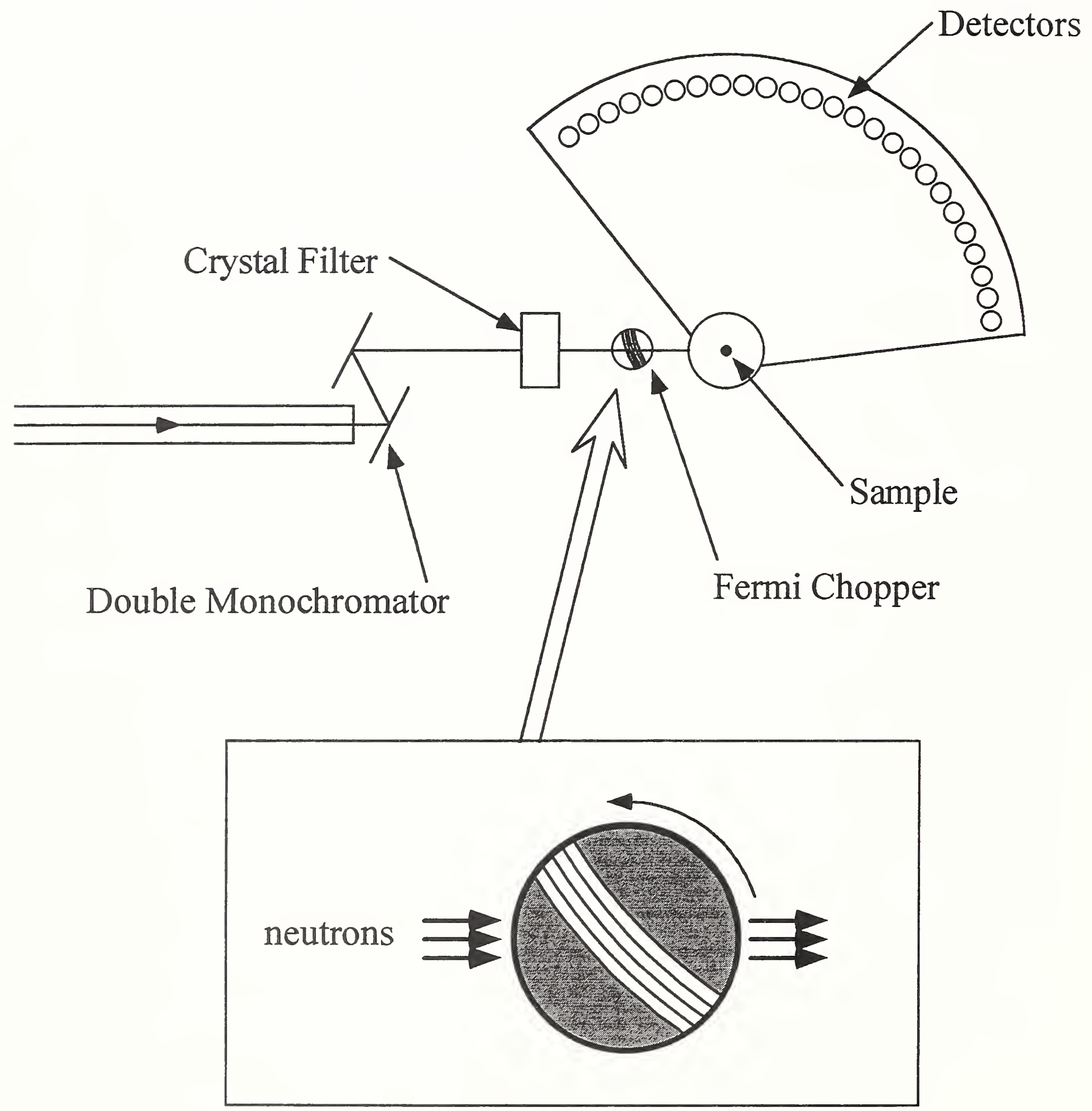

Fig.3 


\section{Disk Chopper Spectrometer (simplified)}

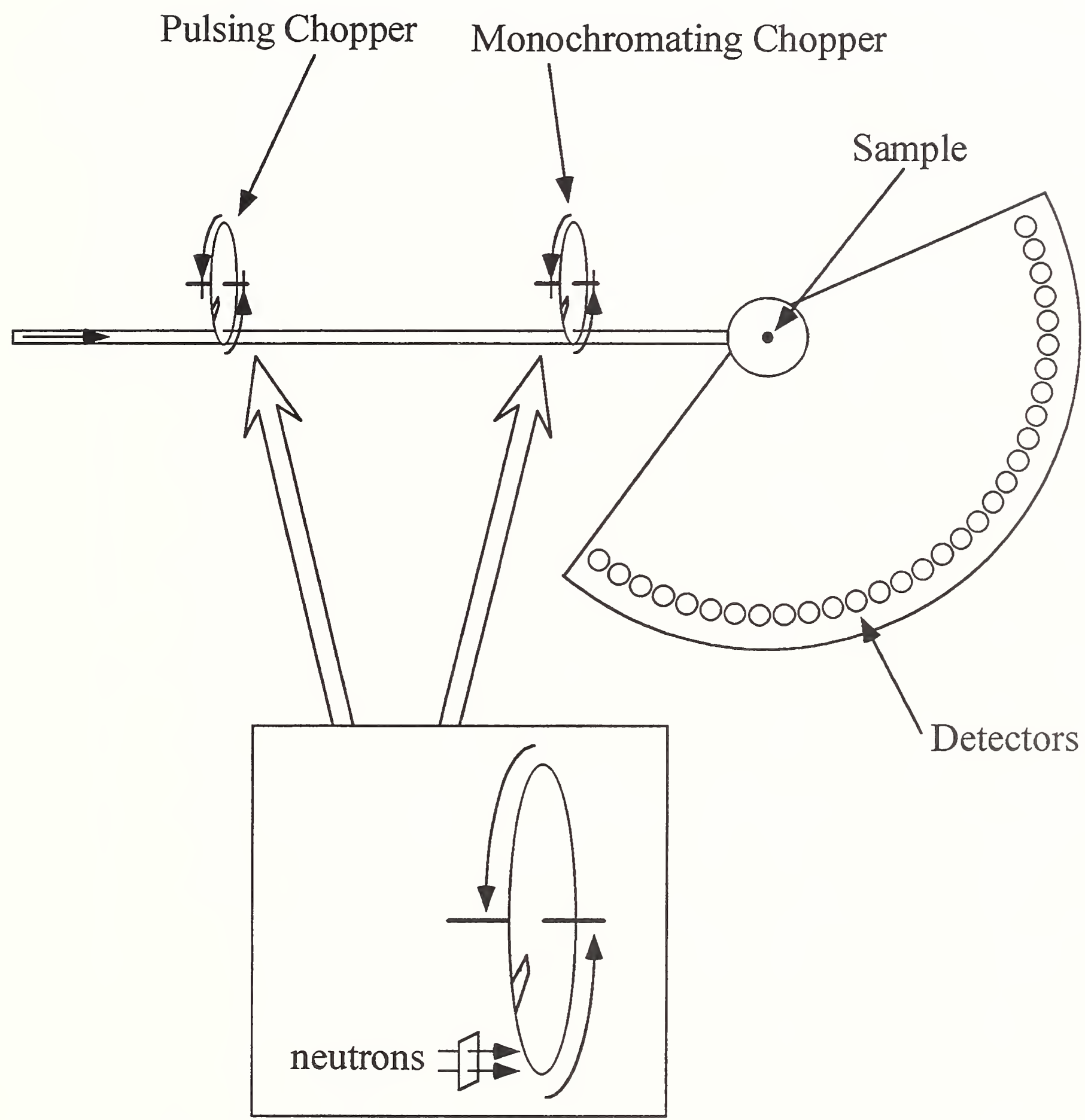

Fig.4 


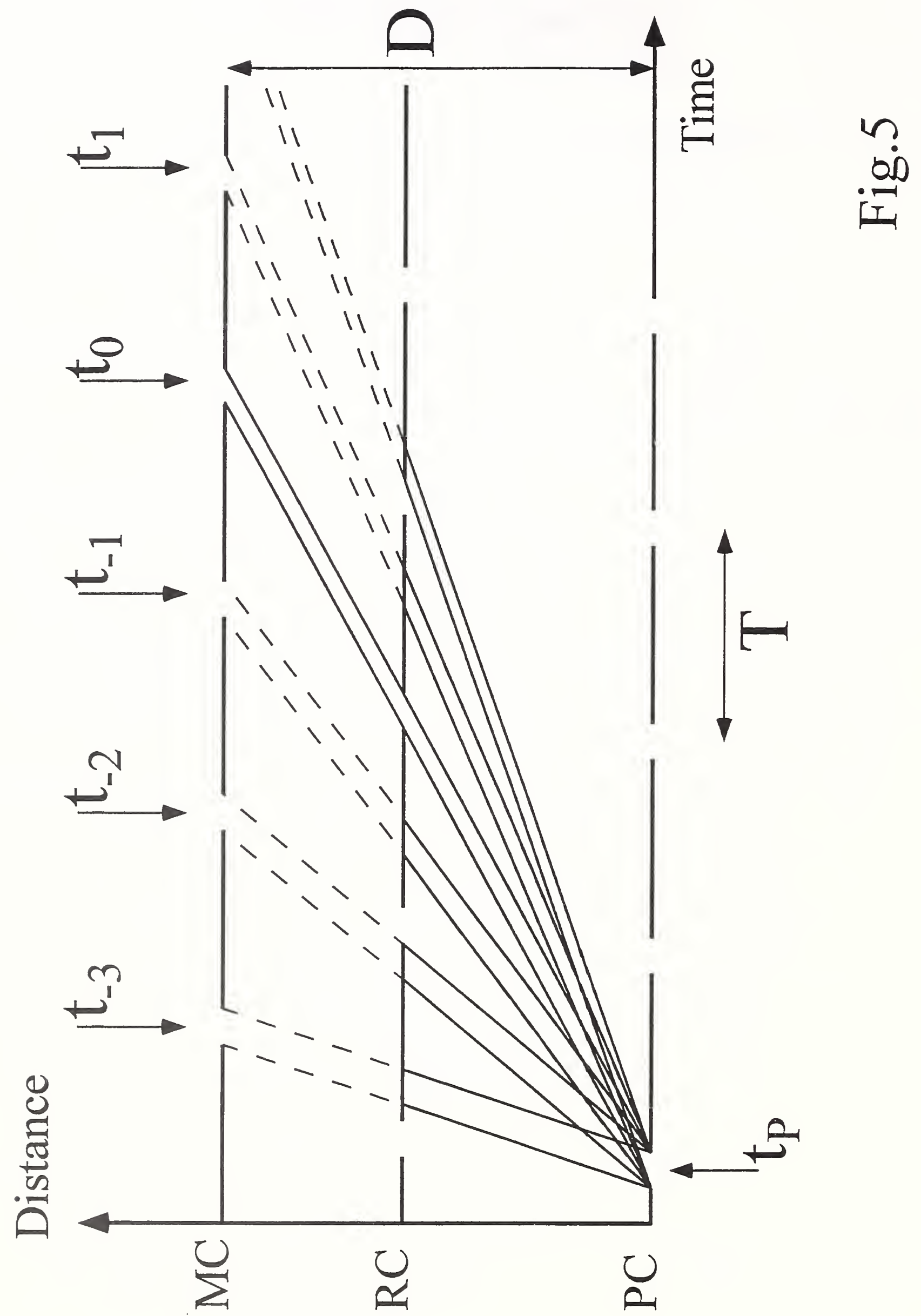


这

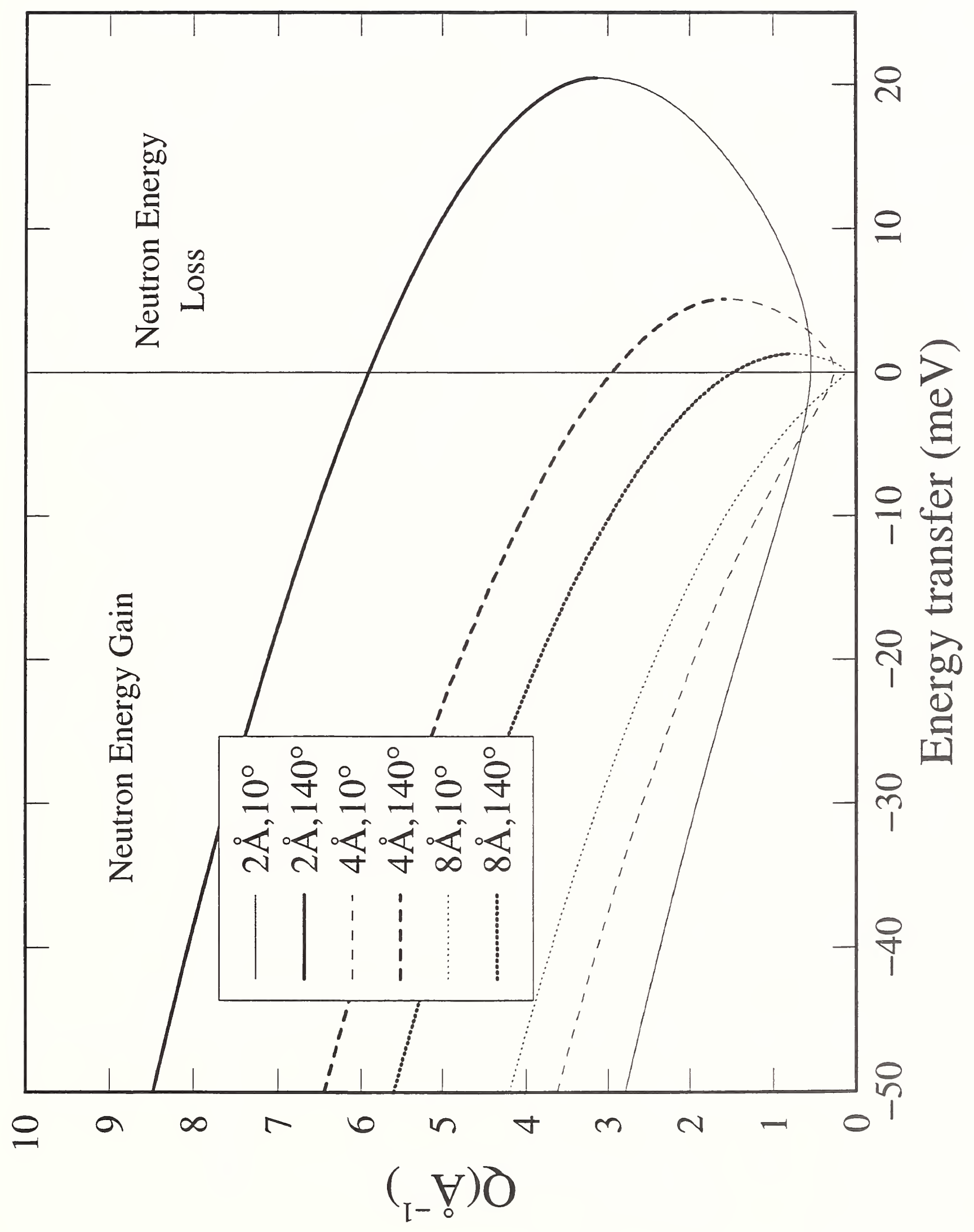




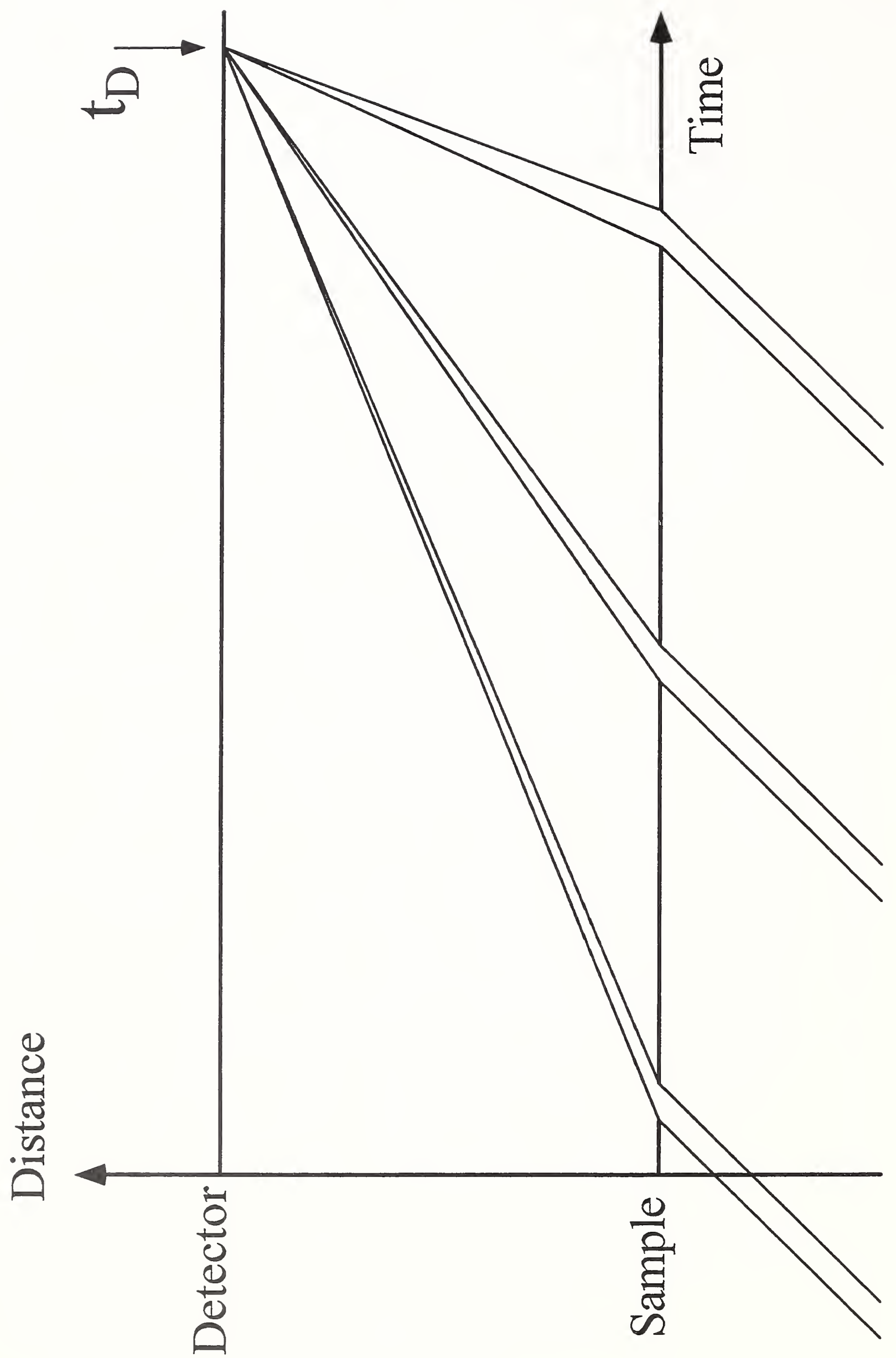




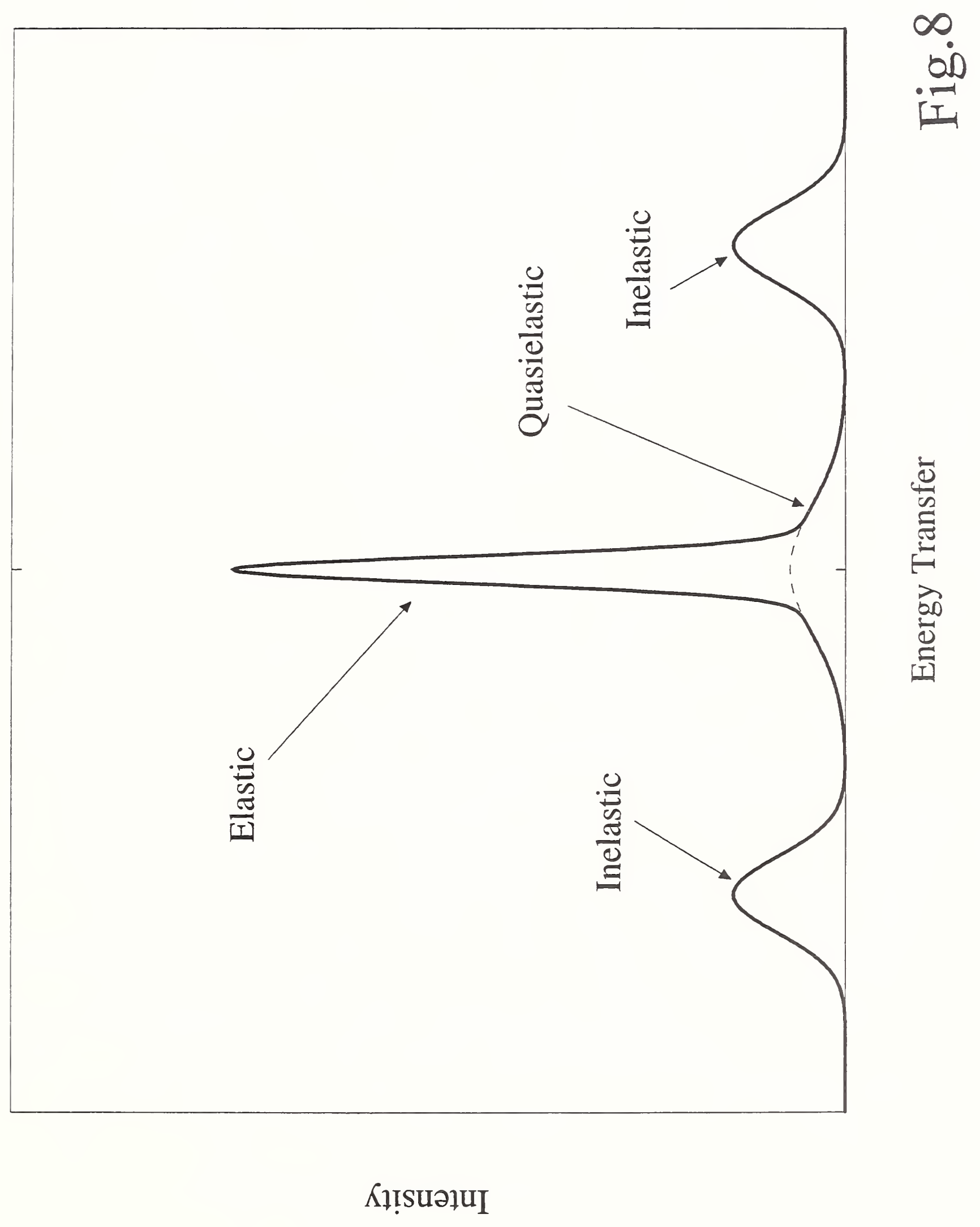




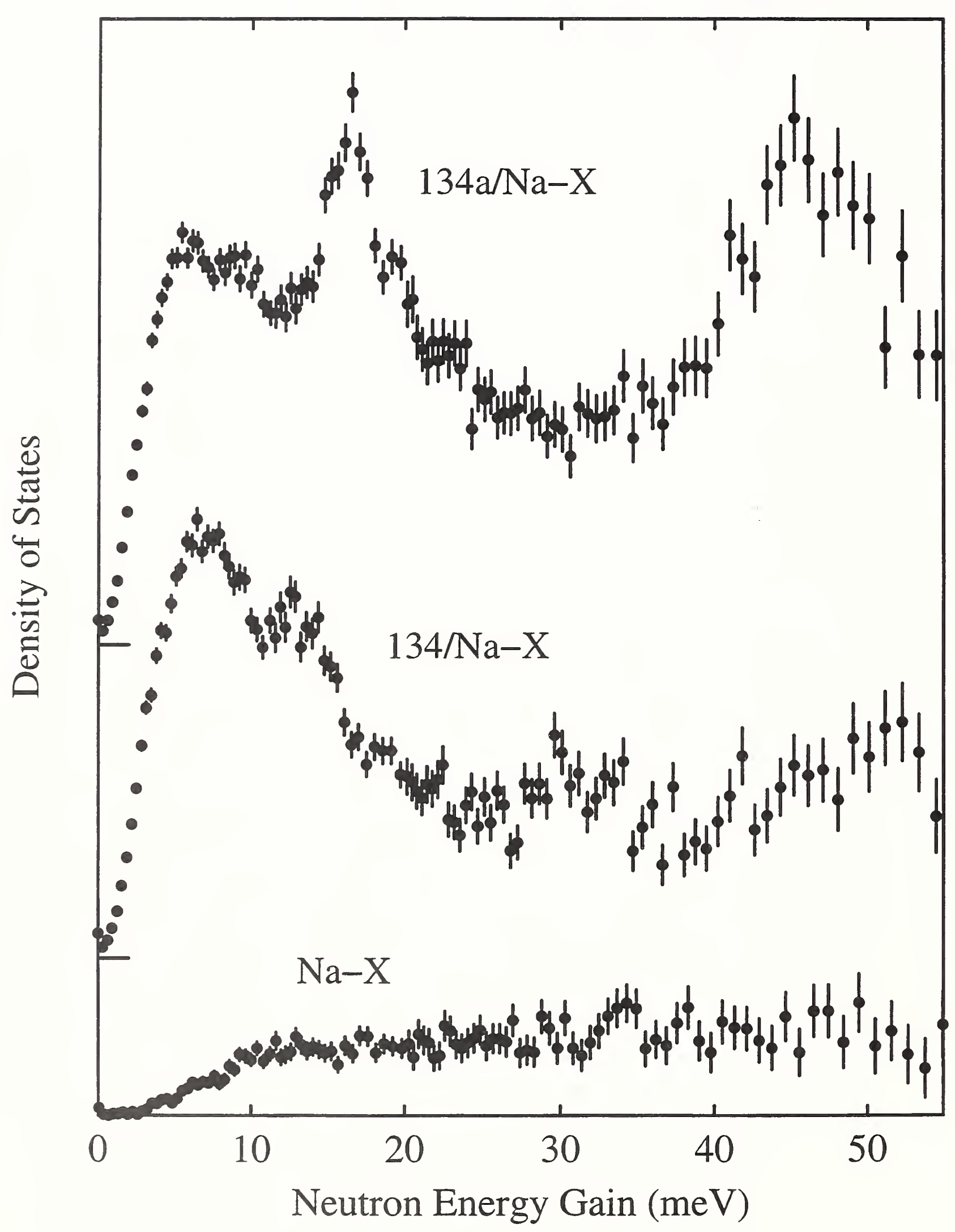

Fig.9 


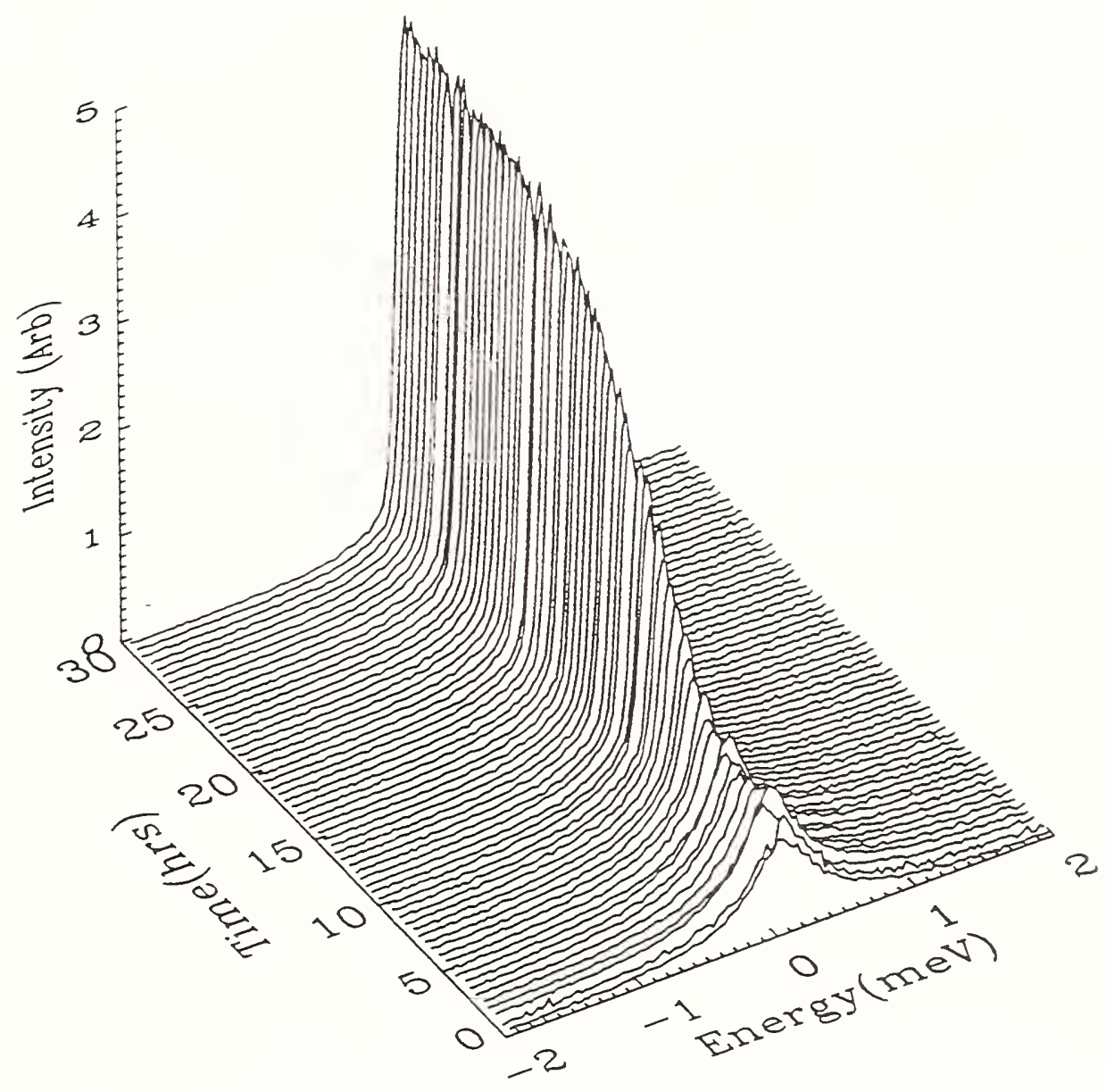

Fig.10 


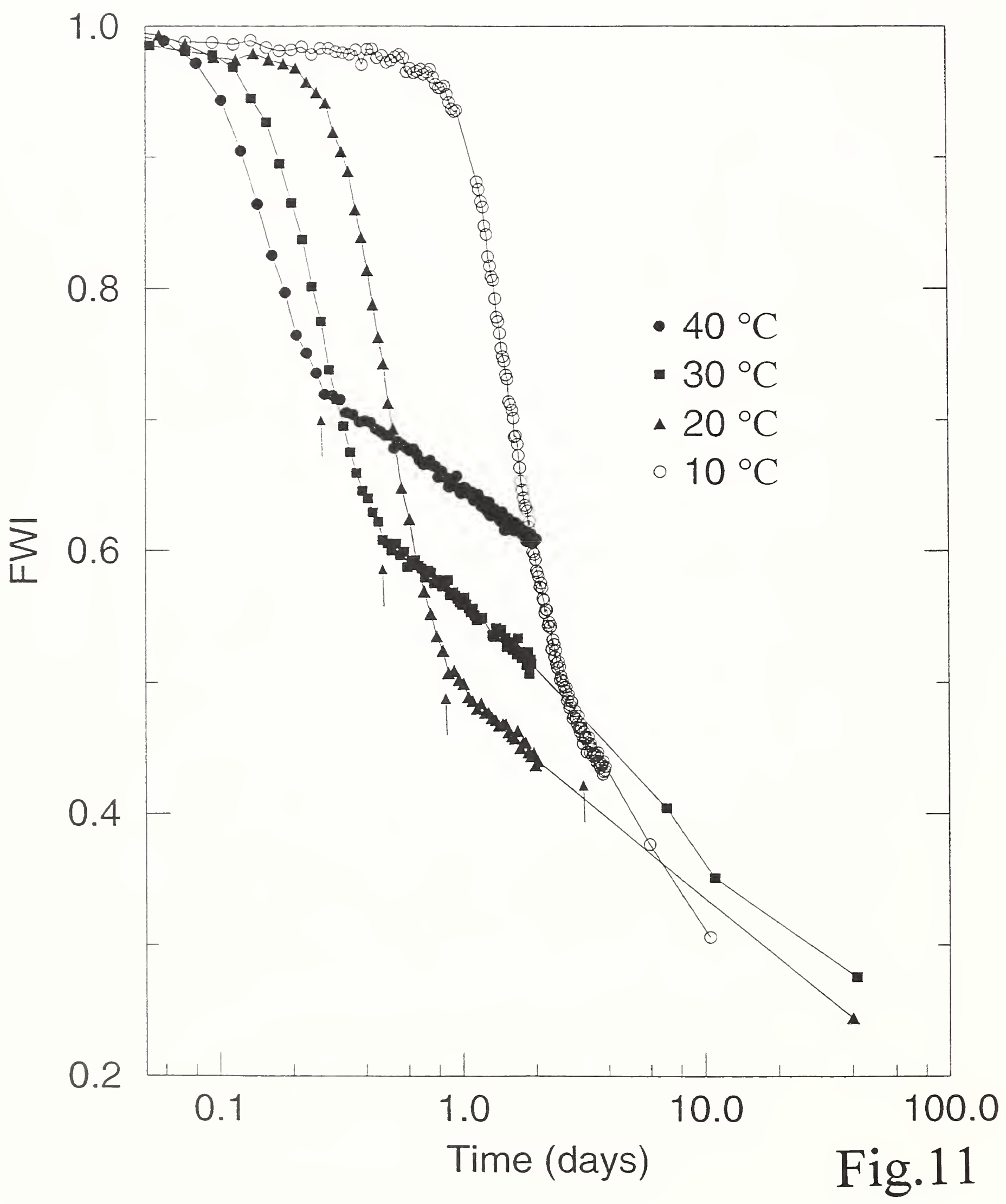




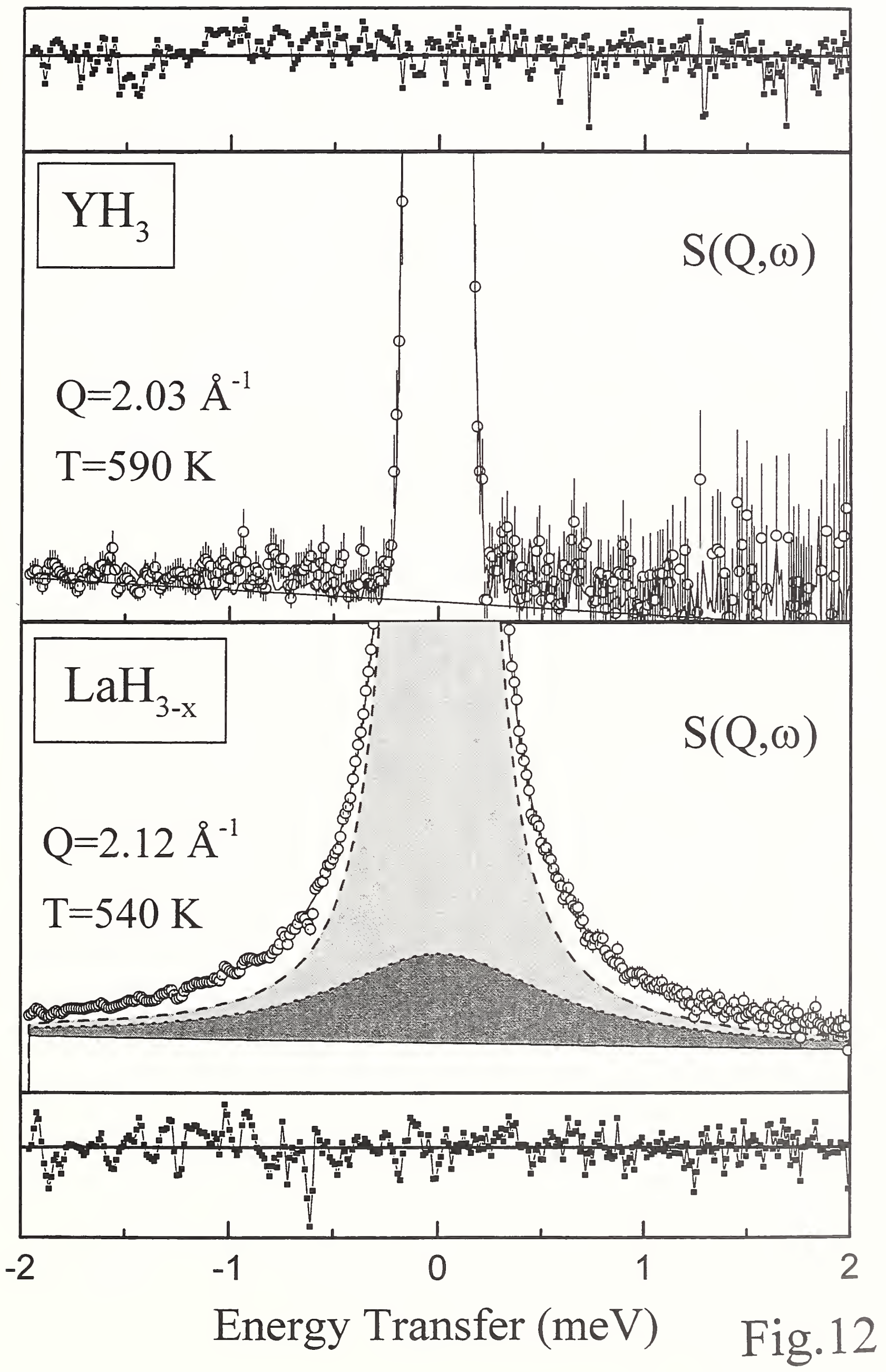




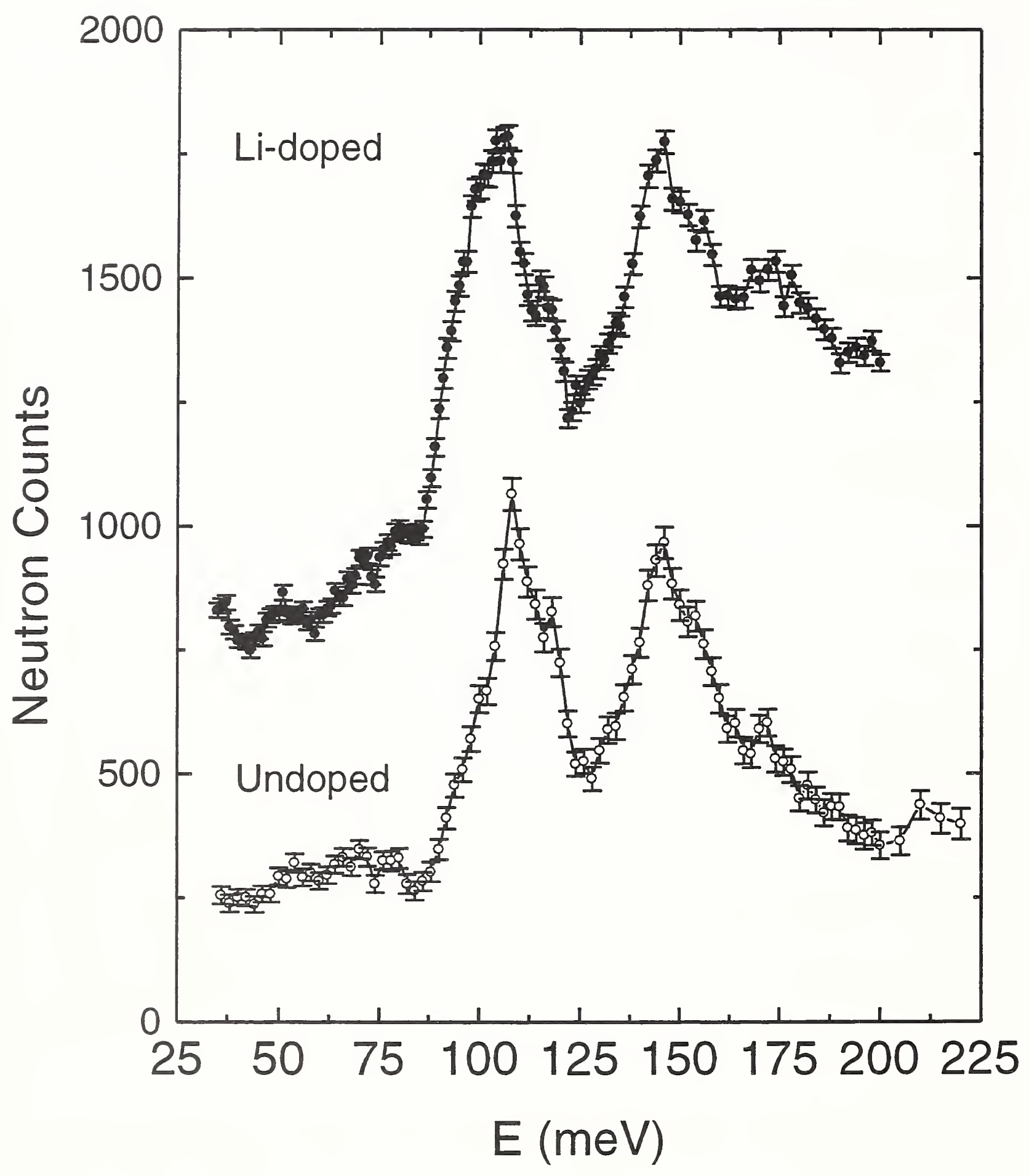

Fig.13 

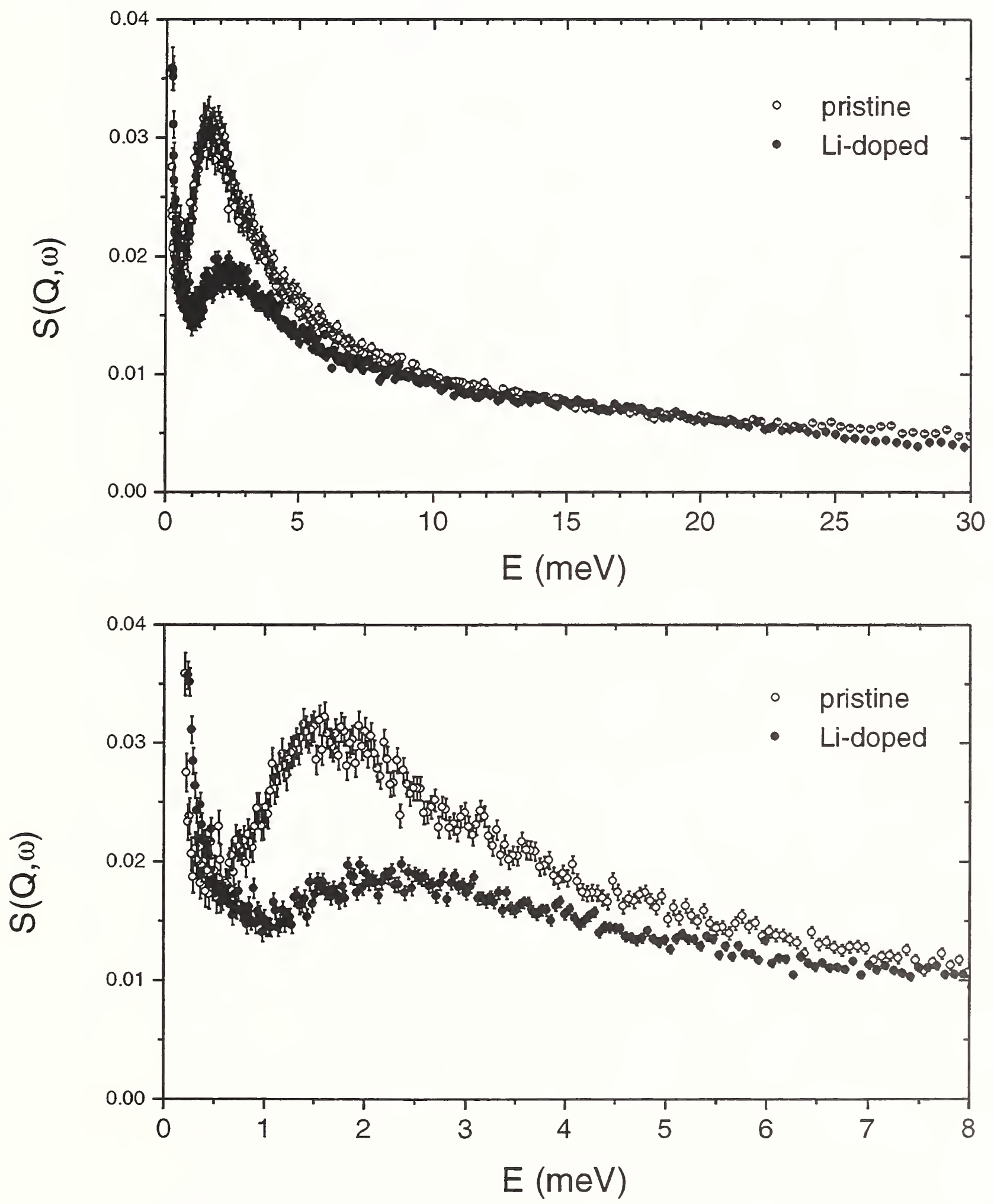

Fig.14 


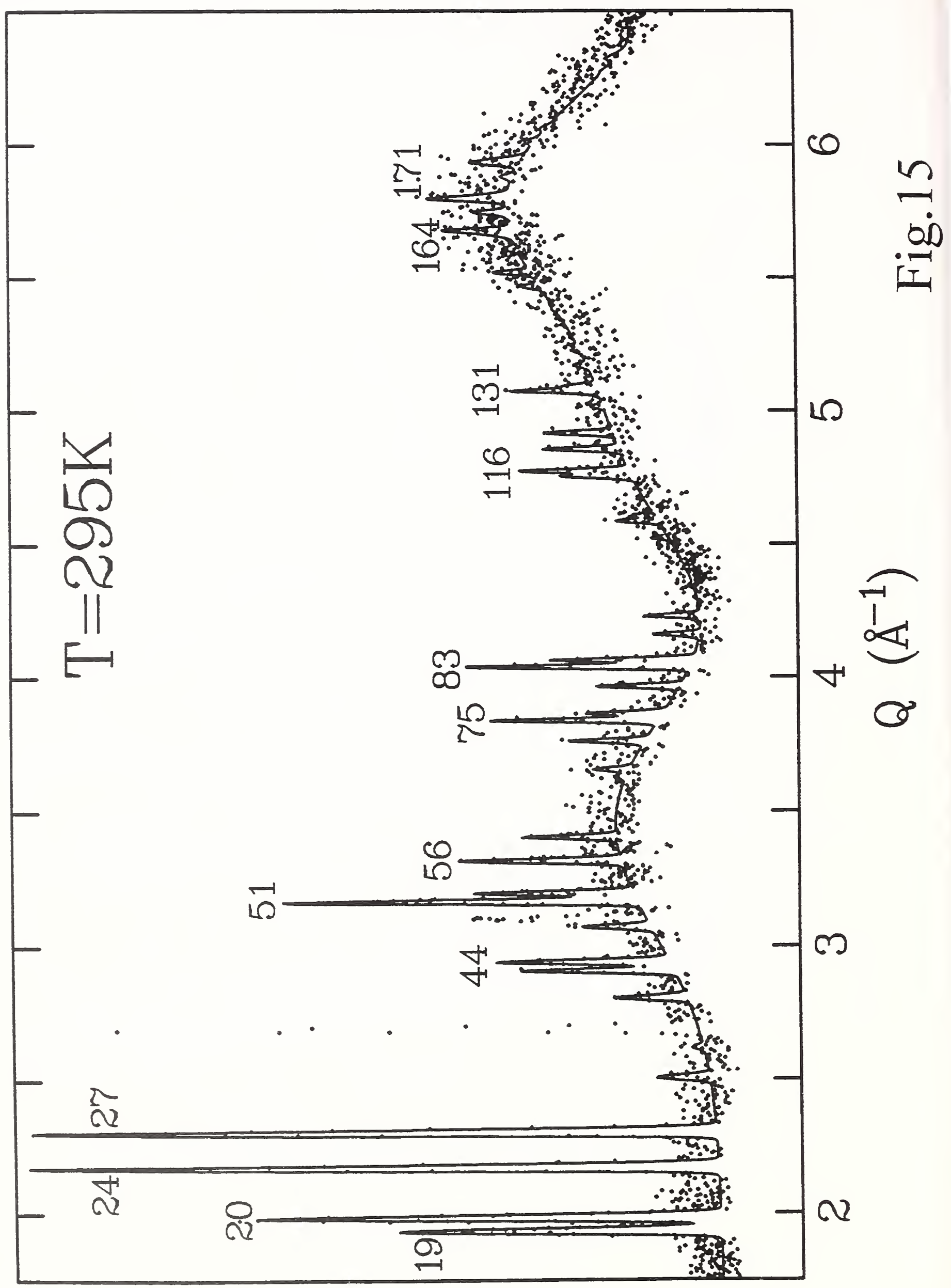




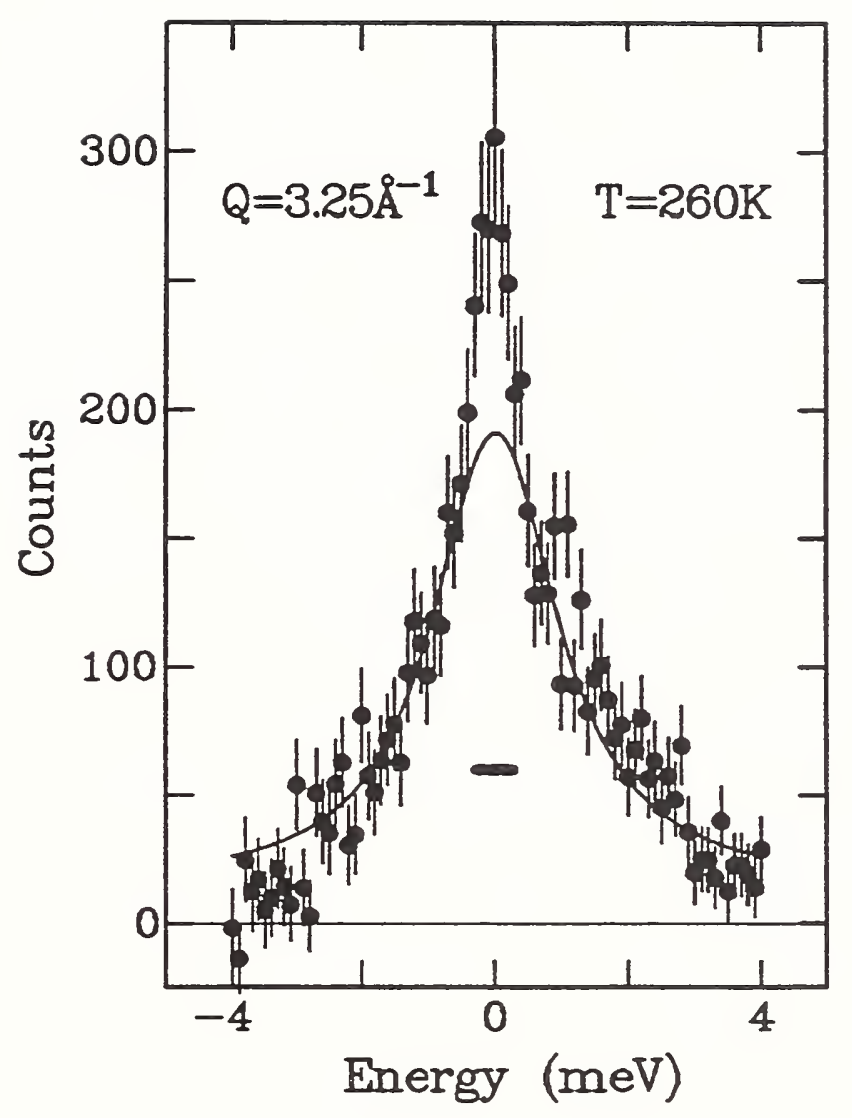

Fig. 16

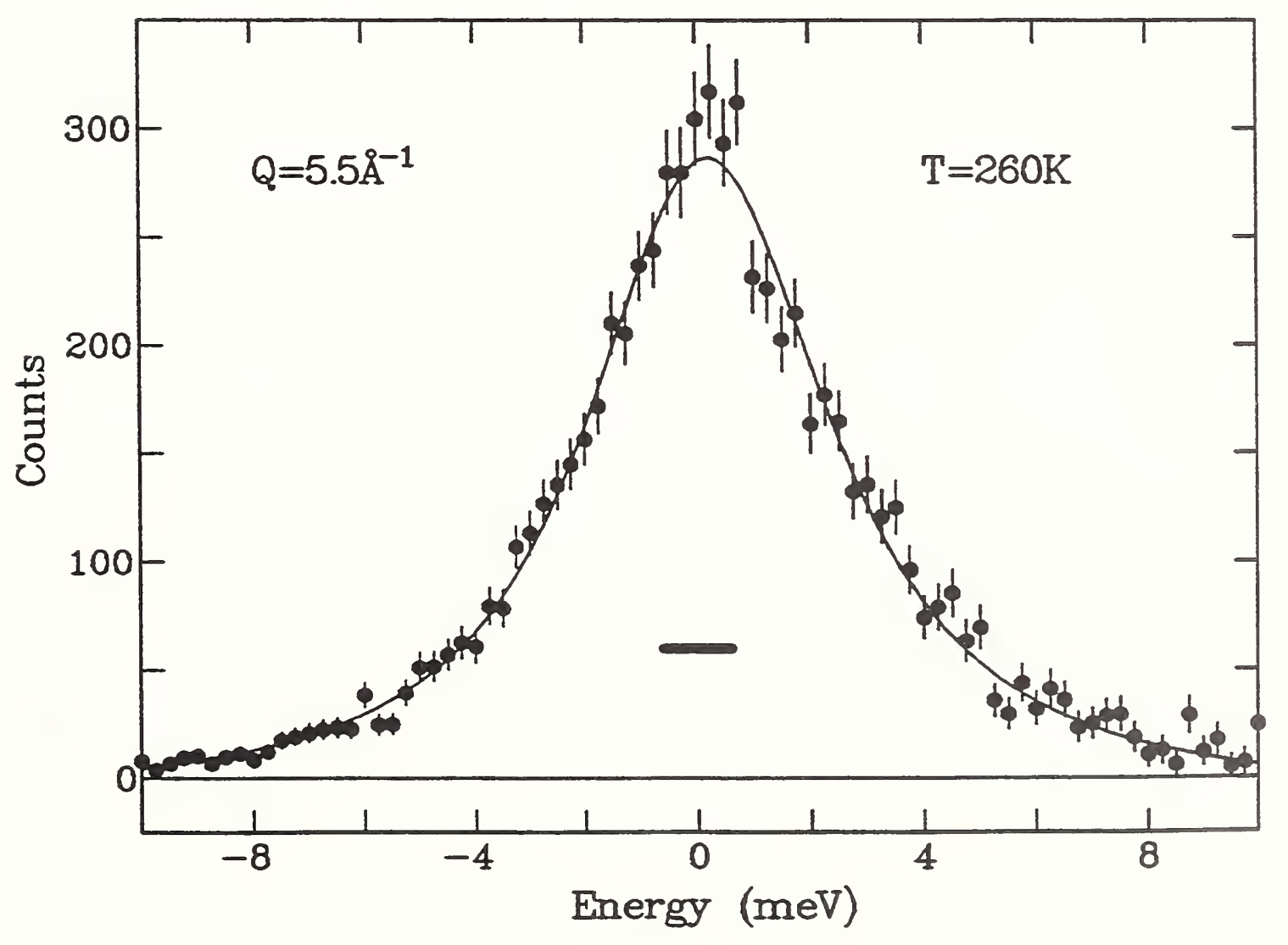




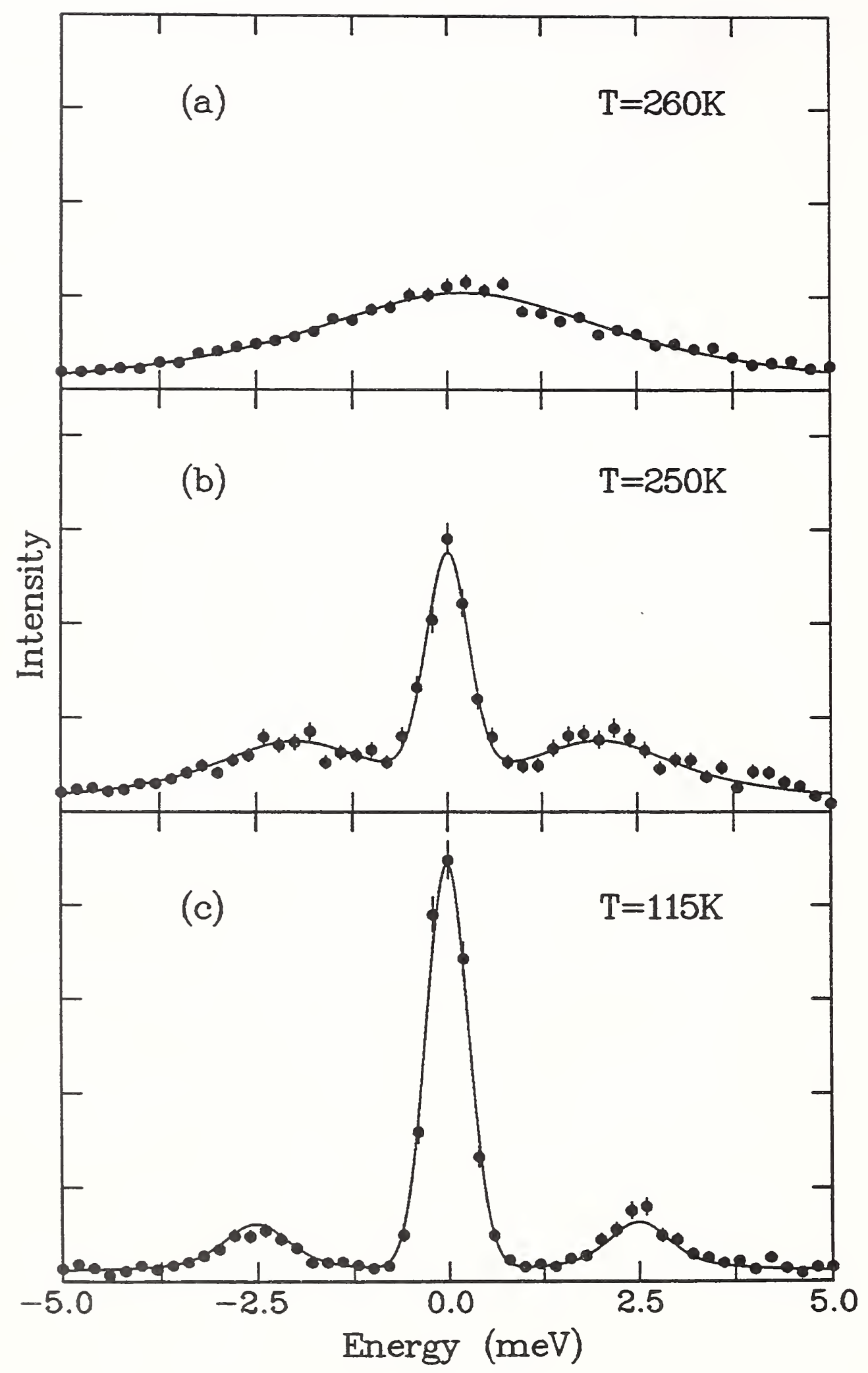

Fig. 17 


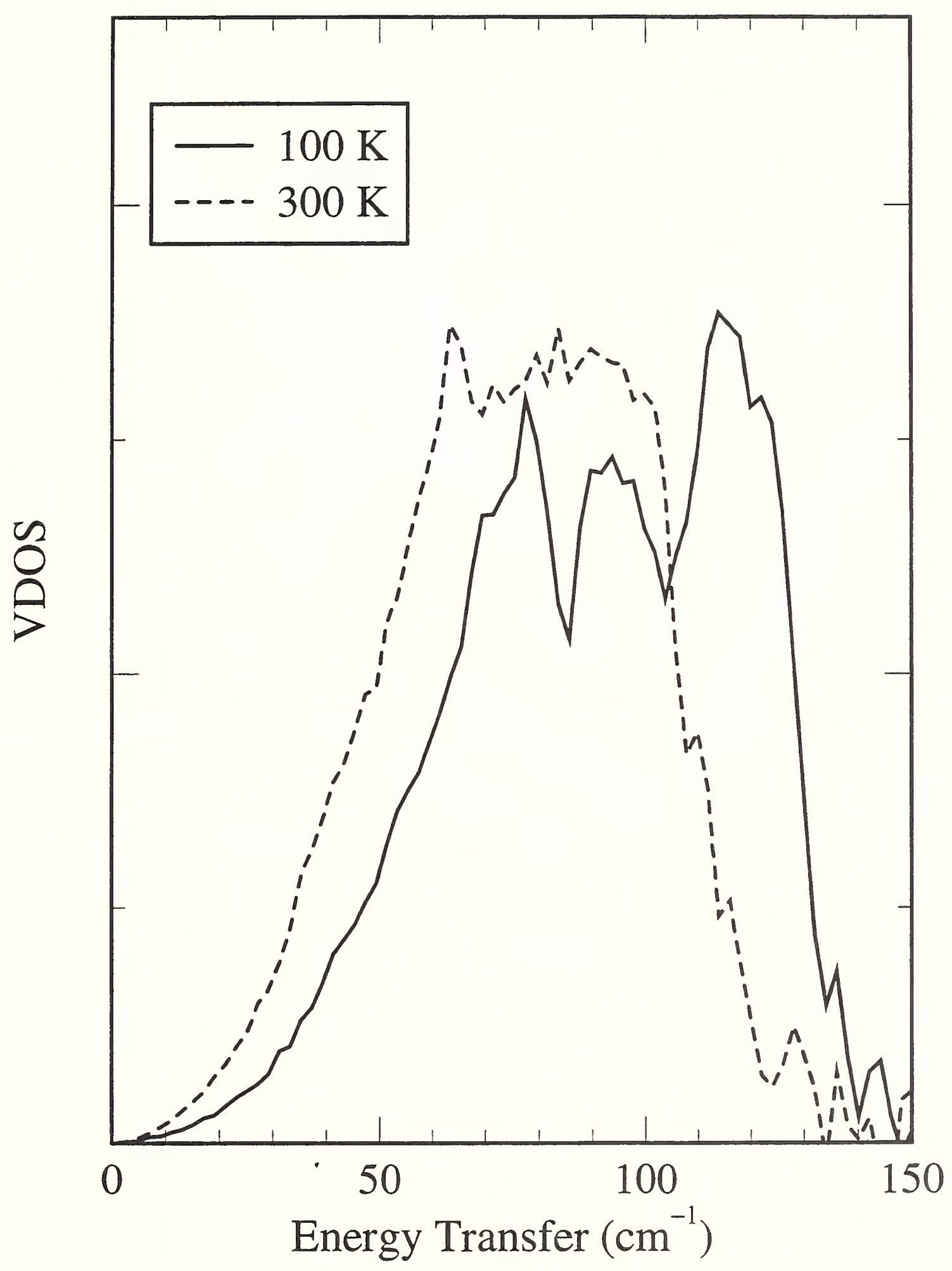

Fig.18 


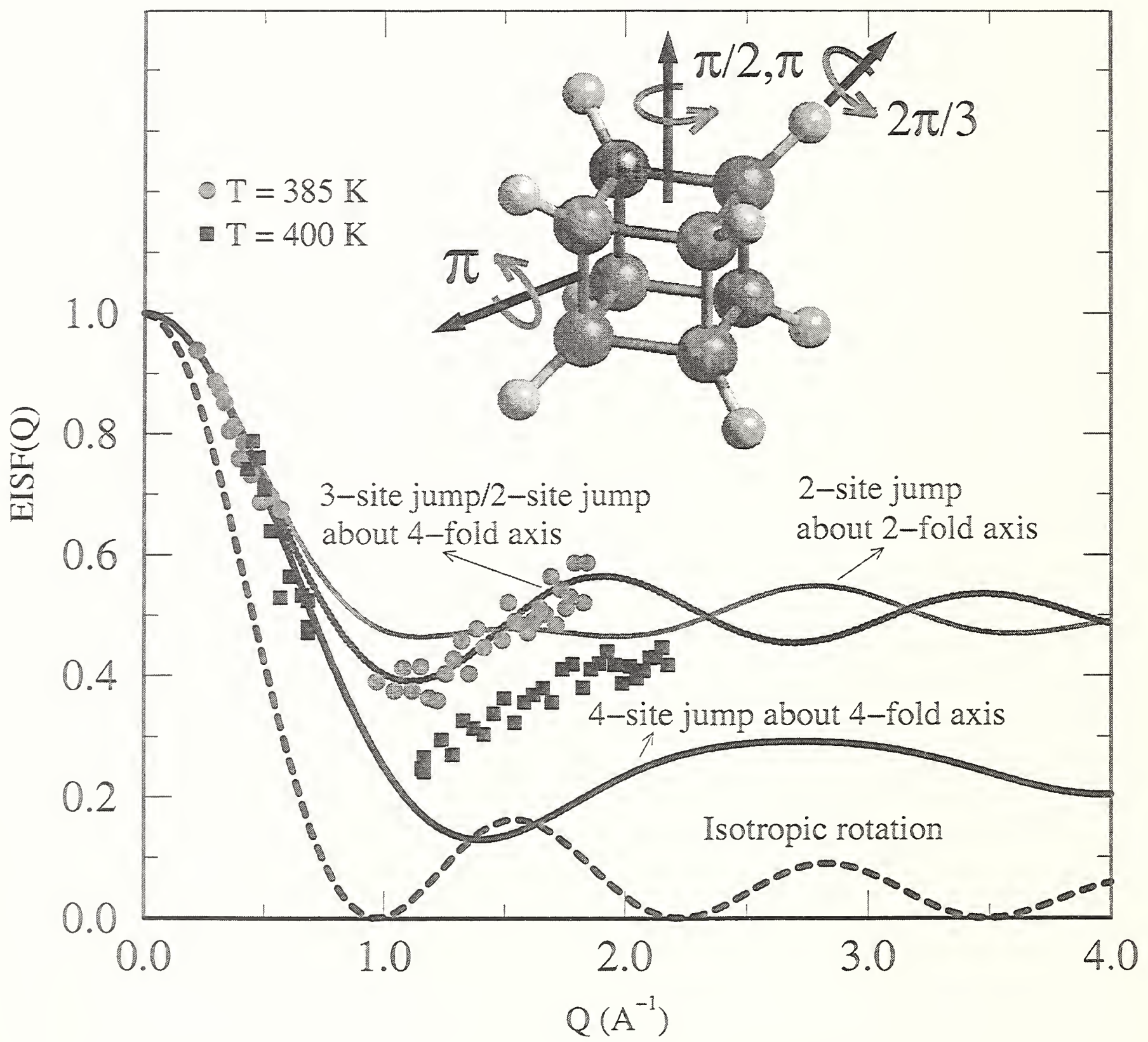

Fig.19 

